Journal of Materials Science

\title{
Glycerol as a green solvent for enhancing the formulation of dextran methacrylate and gellan-based semi-interpenetrating polymer networks --Manuscript Draft--
}

Manuscript Number:

Full Title:

Article Type:

Keywords:

Corresponding Author:
JMSC-D-20-01207R3

Glycerol as a green solvent for enhancing the formulation of dextran methacrylate and gellan-based semi-interpenetrating polymer networks

\section{Manuscript (Regular Article)}

glycerol, green solvent, polysaccharides, semi-interpenetrating polymer networks, gellan gum, dextran methacrylate

Pietro Matricardi, $\mathrm{PhD}$

Universita degli Studi di Roma La Sapienza Facolta di Farmacia e Medicina

Rome, Rome ITALY

\section{Corresponding Author Secondary} Information:

Corresponding Author's Institution:

Universita degli Studi di Roma La Sapienza Facolta di Farmacia e Medicina

\section{Corresponding Author's Secondary} Institution:

First Author:

Nicole Zoratto

First Author Secondary Information:

Order of Authors:

Nicole Zoratto

Roberto Matassa

Elita Montanari

Giuseppe Familiari

Stefania Petralito

Tommasina Coviello

Chiara Di Meo

Pietro Matricardi

Order of Authors Secondary Information:
Abstract: the green chemistry principles. In this work, the ability of glycerol to solubilize high molecular weight polymers and to allow the formation of glycerol-based semi- interpenetrating polymer networks, called 'gly-semi-IPNs' has been investigated. polysaccharides and their mixtures, as well as a DexMA network (obtained by crosslinking in glycerol and in the presence of a photoinitiator) were rheologically mechanical properties of the networks. Interestingly, glycerol was found to deeply influence the mechanical, morphological and optical properties of the formed hydrogels, as well as to improve the crosslinking kinetic of DexMA. Furthermore, and mechanical properties were prepared and characterized.
Funding Information:

Recently, glycerol has been exploited in a number of industrial applications, thanks to its high availability, its low cost and, overall, its peculiar properties which fit well with Compared to water, glycerol deeply affects the structural properties of the biopolymers, especially with reference to their chain flexibility. Among the polysaccharides, dextran methacrylate (DexMA) and gellan gum (Ge) were selected. Glycerol solutions of both characterized to assess the role of the solvent on the chain conformation and on the glycerol leads to more homogeneous mixtures of the two polysaccharides than those observed in aqueous buffers. As such, novel gly-semi-IPNs, with enhanced formulation 
Dear Editor,

Please find enclosed our manuscript entitled "Glycerol as a green solvent for enhancing the formulation of dextran methacrylate and gellan-based semi interpenetrating polymer networks" by Nicole Zoratto et al., which we would like to submit for publication as a full paper in Journal of Materials Science.

In this manuscript, we report the study of glycerol as a green solvent for the development of polysaccharidebased semi-interpenetrate polymer networks (semi-IPNs), based on dextran methacrylate (DexMA) and gellan (Ge). In glycerol such scaffolds showed enhanced properties (i.e. transparency and homogeneity) compared to those prepared in water buffer. DexMA and Ge both in aqueous buffer and glycerol were investigated by rheological analysis, in order to elucidate the solvent effect on the polysaccharide conformations. The "speed up" effect of glycerol on the crosslinking kinetic of DexMA was demonstrated and, hence, exploited to develop DexMA/Ge semi-IPNs. Therefore, these scaffolds were studied in terms of morphological, rheological and dynamo-mechanical properties and compared to those prepared in aqueous buffer.

To the best of our knowledge, it is the first time that glycerol is used as a pure solvent, for developing semiIPNs based on polysaccharides. Furthermore, the capability of glycerol to enhance both the formulation and the properties of these scaffolds suggests its potential application in i.e. 3D printing and biomedicine, making this research appealing to a broad audience.

We confirm this work is original and has not been published elsewhere and is not under consideration by another journal. All authors have approved the manuscript and agree with submission to Journal of Materials Science.

We wish to send the present manuscript to the following reviewers:

1) Carmen Alvarez-lorenzo, carmen.alvarez.lorenzo@usc.es, Santiago de Compostela University, Faculty of Pharmacy.

Expertise in polymer chemistry, hydrogel formulation and polysaccharides.

2) Mario Grassi, mario.grassi@dia.units.it, University of Trieste, Faculty of Engineering and Architecture.

Expertise in rheology, polymer chemistry and hydrogel development.

3) Amir Sheikhi, sheikhi@psu.edu, Faculty of Chemical Engineering.

Expertise in biomaterials, hydrogel formulation and characterization.

Best regards

Pietro Matricardi 
COMMENTS FOR THE AUTHORS:

\section{Comments from editor:}

Before sending a revised version following reviewers comments, please check carefully the following points:

[ ] Title is grammatically correct and sufficiently general to attract citations

[ ] Text in figures and schemes is spelled correctly

[ ] Any machine-generated data bars have been replaced with scale markers on SEM images.

[ ] You provided a graphical abstract representative of your work and attracting reader attention

The authors thank the Editor for the comments. The above points have been revised accordingly.

\section{Graphical abstract}

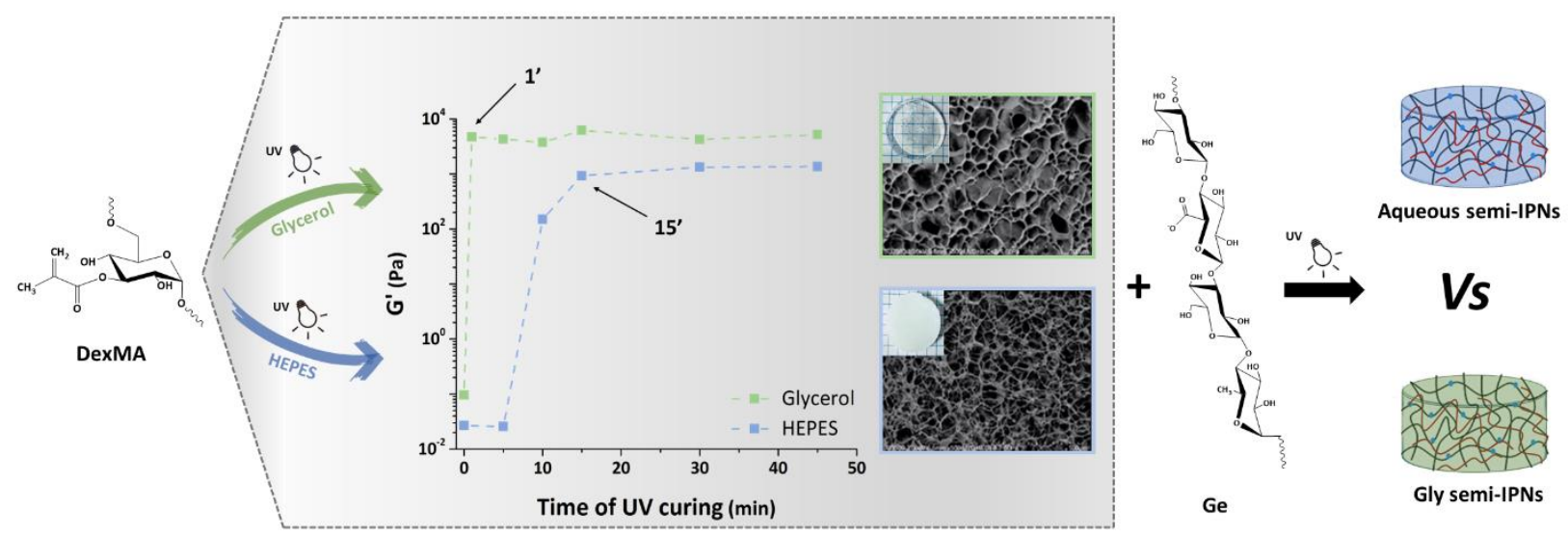

\section{Comments from reviewers:}

Reviewer \#1: (1) Please add a schematic diagram of gel formation, highlighting clearly the role of glycerin in it.

The authors thank the Reviewer for the suggestion. We added the following schematic diagram of the gel formation in the revised manuscript, highlighting the role of glycerine. Moreover, the following sentence has been added to the main manuscript (section 3.1.) in order to clarify this concept.

"Moreover, glycerol may form strong hydrogen bonds with Ge chains thanks to its $\mathrm{OH}$ groups, thus leading to a further reduction of the intermolecular interactions among Ge chains". 
a)

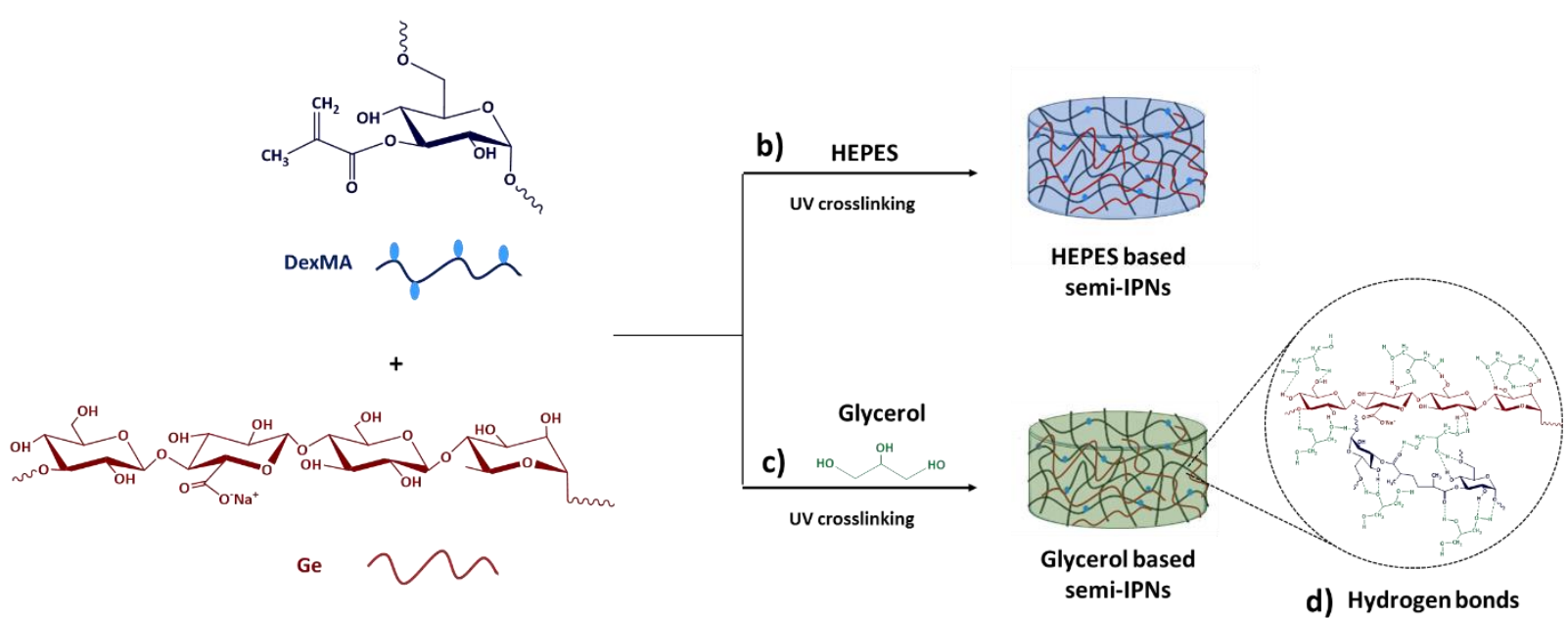

Fig 5: Scheme of DexMA-Ge semi-interpenetrating polymer network formation. a) Structures and schematic representation of DexMA and Ge. b) Formation of DexMA-Ge semi-IPNs in pure HEPES after UV curing. c) Formation of gly-semi-IPNs in pure glycerol after UV curing. d) Schematic representation of the possible interactions via hydrogen bonds among glycerol, DexMA and Ge in gly semi-IPNs.

\section{(2) Regarding 3D printing, whether the gel state formed by single glycerin as a solvent in Figure 4a can ensure the integrity of printing?}

The authors thank the Reviewer for the comment. The scaffold integrity can be ensured in pure glycerol, since the crosslinking of the methacrylate moieties of DexMA was confirmed by replacing the glycerol with HEPES in the network after UV curing, thus suggesting the retention of both, structure and shape. Specifically, after the chemical crosslinking of DexMA in glycerol $(5 \% \mathrm{w} / \mathrm{w})$, the gel was poured in $50 \mathrm{~mL}$ of HEPES $(0.05 \mathrm{M}, \mathrm{pH}=7.4)$ and the external solvent was then removed and replaced several times by fresh HEPES, in order to completely remove glycerol. After $48 \mathrm{~h}$ washing, the starting shape of the network was completely retained.

(3) Regarding 3D printing, we know that color and light will affect each other. So, as far as the gel formation method in this article is concerned, is it possible to do color 3D printing?

The authors thank the Reviewer for the comment. Coloured 3D printing gels can be made by using dyes or coloured molecules that do not interfere with the radical crosslinking process initiated by the photoinitiator I-2959.

In this perspective, we added Vitamin B12 (VitB12, red) to DexMA solutions or DexMA/Ge mixtures and the systems were crosslinked in the presence of I-2959 as a photoinitiator. Figure R1 shows the picture of the chemically crosslinked network (formed by $5 \%$ w/w DexMA and $0.75 \%$ w/w Ge) loaded with VitB12 as a dye. The figure shows that no changes in the network shape or in the dimensions of the network occur after the loading of VitB12 molecules and the UV curing. Moreover, after the addition of the VitB12, the gel keeps the original optical transparency. 


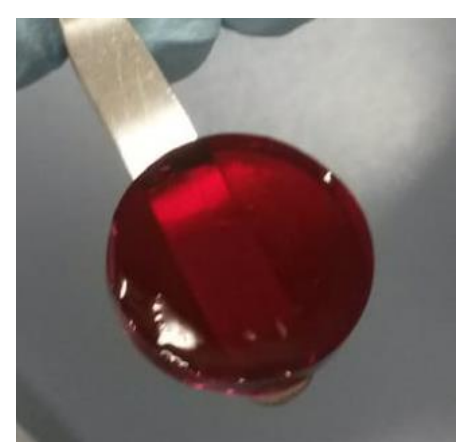

Fig R1: Image of 5\% w/w DexMA/0.75\% w/w Ge network loaded with VitB12 in glycerol, after UV curing performed in the presence of I-2959 as a photoinitiator.

(4) Some related work should be cited such as Journal of Materials Chemistry B, 2019, 7, 2577-2587; Journal of Agricultural and Food Chemistry, 2018, 66, 10479-10489; Molecular Pharmaceutics, 2017, 14, 431-440; Materials Science \& Engineering C, 2017, 75, 487-494, ACS Biomaterials Science \& Engineering, 2015, 1, 12871299.

The authors thank the Reviewer for the comment. The appropriate references have been added to the main manuscript.

(5) The article mentions biological applications, but lacks corresponding proofs, and the mechanism of glycerin is not clearly stated, so please prove that the materials in this article can be used in biological applications.

The authors thank the Reviewer for the comment and agree. Very preliminary cell viability tests have been performed by using the human keratinocyte cell line (HaCaT). Cells were seeded on the top of 5\% w/w DexMA/0.75\% w/w Ge disk scaffolds and the systems were incubated at $37^{\circ} \mathrm{C}$ in $5 \% \mathrm{CO}_{2}$ for 24,48 and $72 \mathrm{~h}$. The cell viability was then checked by adding MTS reagent (Figure R2 a) and the obtained absorbance values (at each time point) were normalised to the absorbance recorded at $24 \mathrm{~h}$ (Figure R2 b). Results show a significant cell growing within $72 \mathrm{~h}$, suggesting that the gels are not toxic to the cells. In fact, as shown in Figure a), at $72 \mathrm{~h}$ the O.D. values were higher than those at $24 \mathrm{~h}$, confirming the cell proliferation over the time, hence the low cytotoxicity of the semi-IPNs, both in HEPES and in glycerol. Furthermore, the scaffolds prepared in glycerol show a significant higher proliferation rate than those prepared in HEPES, after $72 \mathrm{~h}$. This might be related to the effect of the solvent on the pore structure and on the pore size distribution in the two networks. Further biological studies will be carried out in the next work. 
a)

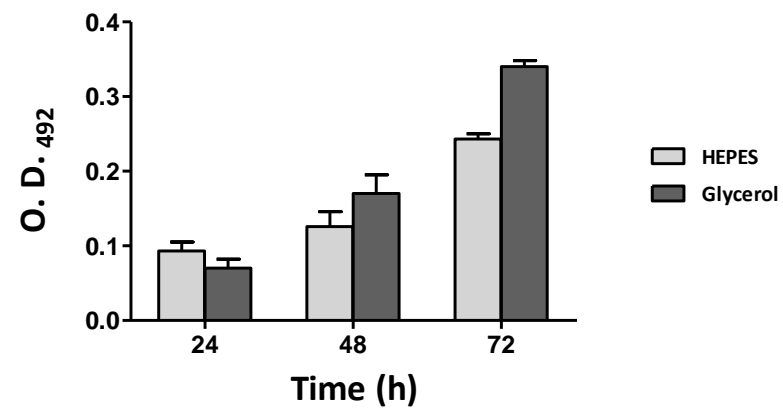

b)

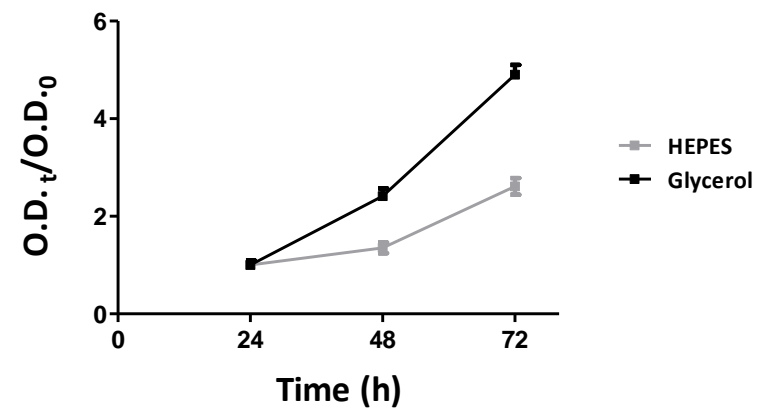

Fig R2: Cell viability assay: MTS test. a) Cell proliferation was determined with the MTS assay, at 24, 48 and $72 \mathrm{~h}$. HaCaT cells were seeded on semi-IPNs formed by $5 \%$ w/w DexMA and 0.75\% w/w Ge, prepared both in HEPES and glycerol. b) The absorbance values (O.D.492) were collected at each time point and then normalized to those recorded at $24 \mathrm{~h}$. The results represent the means \pm standard deviation with $n=3$ for each error bar.

(6) In the case where Ge has a positive effect on the mechanical properties of the final formed DexMA/Ge gly-semi-IPNs, does it have an effect on its pore structure?

The authors thank the Reviewer for the comment. The porosity of 5\% w/w DexMA network in glycerol or HEPES was investigated by VP-SEM, with the main target to understand the effect of glycerol on the morphology of DexMA network. The obtained images show that glycerol significantly affects the scaffold porosity in terms of pore size distributions and interconnections. Regarding to the effect of Ge on the pore structure, it was observed that the gel mesh size (६) of the semi-IPNs prepared in glycerol, obtained by applying the Flory theory and using the G' values at $1 \mathrm{~Hz}$, were not dependent on the presence and on the concentration of Ge, evidencing mesh size values around $13 \mathrm{~nm}$ in all the cases. The next step could be to investigate in details the effect of the Ge concentration on the network pore structure and pore size distribution by using VP-SEM.

(7) Please add the influence trend of Ge concentration on the mechanical properties of DexMA / Ge gly-semi$\underline{\text { IPNS. }}$

The authors are thankful to the Reviewer for the comment. In order to better clarify this point, the main manuscript was revised and improved (section 3.4), accordingly. 
Reviewer \#3: This paper, dealing with the use of glycerol as a green solvent for the formulation of SIP polymeric particles, is interesting and well written. Accordingly, only minor issues arose from its careful reading. In details:

1) In order to characterize their systems, the authors make use of SEM that implies (at least judging from Figure 4) solvent removal from the hydrogel. Would it not be more appropriate using an Environmental SEM that does not imply solvent removal that could be responsible for artifacts?

The authors thank the Referee for the useful comment. As suggested by the Reviewer, ESEM is the most appropriate technique to investigate the network morphology, since it does not imply the solvent removal, avoiding artefacts. However, the VP-SEM (5-650 Pa, Hitachi SU-3500) used in our work allows to study the samples in their hydrated state since the experiments were performed following the saturated vapour pressure curve. In detail, our VP-SEM is equipped with a Peltier cool-stage control which allows the stabilization of the hydrated sample with the correlative pressure/temperature at $60 \mathrm{~Pa} /-25^{\circ} \mathrm{C}$. This experimental procedure ensures the high-resolution imaging of hydrated specimens under varying pressure conditions. Furthermore, the low deposition rates combined with specimen cooling, lead to a damage reduction of the sample, which is thermally fragile. The authors have modified the experimental procedures in the main manuscript (section 2.2.5), as reported below:

\section{“2.2.5. Variable Pressure Scanning Electron Microscopy}

The hydrogel morphology was studied using a variable pressure scanning electron microscope (VP-SEM, Hitachi SU3500). Cross-sectioned hydrated samples were observed without conductive coating. VP-SEM combined with Peltier coolstage control allowed high-resolution imaging of hydrated specimens under varying pressure conditions as well as limiting water vapour loss through the control of stage temperature. A thin film of distilled water was deposited onto the hydrated samples at room temperature, in order to limit the water-loss during low vacuum procedures. By controlling the chamber pressure and the cooling temperature, the collapse of the surface pores was avoided. All the samples were observed at an accelerating voltage of $8 \mathrm{KV}{ }^{28}$, considering a working distance between 5 and $6 \mathrm{~mm}$ and a correlative pressure/temperature stabilized at $60 \mathrm{~Pa} /-25{ }^{\circ} \mathrm{C}$ before the measurements. The pore size and area of hydrogels were quantified using Fiji software (NHI-USA)".

2) The authors say that ".... considering the potential biomedical applications of these scaffolds". Thus, my question is about the final destination of their system. Are these systems supposed to be used as swollen gels or are they supposed to be used after solvent removal as depicted in Figure 4?

The authors are thankful to the Reviewer for the interesting comment. The systems could be potentially used both as swollen gels or after solvent removal. So far, the viability of epithelial cells ( $\mathrm{HaCaT}$ ) has been investigated on the swollen gels. Preliminary MTS tests were performed on the 5\% DexMA/0.75\% Ge semi-IPNs, showing that such networks were not toxic to the cells as they did not interfere with the cell metabolism over the whole period of time investigated (72 h).

Further biological experiments are necessary to elucidate the specific biomedical application of these systems. 

theory and the value of $\mathrm{G}^{\prime}$ at $\left.1 \mathrm{~Hz}\right)$ ?

The authors thank the referee for the stimulating comment. The gel mesh sizes ( $\xi)$ were estimated by applying the Flory theory (and G' values at $1 \mathrm{~Hz}$ ), showing values of about $13 \mathrm{~nm}$ for the samples prepared in glycerol, regardless of the presence of Ge chains. On the other side, the samples prepared in HEPES showed $\xi$ values significantly dependent on the presence and on the concentration of Ge (ranging from about $16 \mathrm{~nm}$ for the crosslinked DexMa to about $7 \mathrm{~nm}$ for the semi-IPN with Ge 2\%). In the experimentally explored conditions, a straightforward correlation between rheology and SEM images cannot be applied for different reasons, mainly because the dimensional ranges explored by the microscopy technique and by the rheology measurements are different. 


\title{
Glycerol as a green solvent for enhancing the formulation of dextran methacrylate and gellan-based semi-interpenetrating polymer networks
}

\author{
Nicole Zoratto, ${ }^{\mathrm{a}}$ Roberto Matassa, ${ }^{\mathrm{b}}$ Elita Montanari, ${ }^{\mathrm{a}}$ Giuseppe Familiari, ${ }^{\mathrm{b}}$ Stefania Petralito, ${ }^{\text {a }}$ \\ Tommasina Coviello, ${ }^{a}$ Chiara Di Meo ${ }^{\mathrm{a}}$ and Pietro Matricardi ${ }^{*}$ \\ a Department of Drug Chemistry and Technologies, Sapienza, University of Roma, P.le A. Moro 5, 00185 Roma, Italy. \\ ${ }^{b}$ Department of Anatomical, Histological, Forensic and Orthopaedic Sciences, Section of Human Anatomy, Sapienza University of \\ Rome, Via A. Borelli 50, 00161 Rome, Italy. \\ e-mail: nicole.zoratto@uniroma1.it; orcid 0000-0002-4642-8227 \\ roberto.matassa@unirma1.it; orcid 0000-0002-6336-4587 \\ elita.montanari@uniroma1.it; orcid 0000-0002-0551-5113 \\ giuseppe.familiari@uniroma1.it; orcid 0000-0002-3456-1434 \\ stefania.petralito@uniroma1.it; orcid 0000-0002-0281-4097 \\ tommasina.coviello@uniroma1.it; orcid 0000-0003-3356-7567 \\ chiara.dimeo@uniroma1.it; orcid 0000-0002-3531-6107 \\ *Corresponding author: pietro.matricardi@uniroma1.it; orcid 0000-0003-2086-911X
}

\begin{abstract}
Recently, glycerol has been exploited in a number of industrial applications, thanks to its high availability, its low cost and, overall, its peculiar properties which fit well with the green chemistry principles. In this work, the ability of glycerol to solubilize high molecular weight polymers and to allow the formation of glycerol-based semiinterpenetrating polymer networks, called 'gly-semi-IPNs' has been investigated. Compared to water, glycerol deeply affects the structural properties of the biopolymers, especially with reference to their chain flexibility. Among the polysaccharides, dextran methacrylate (DexMA) and gellan gum (Ge) were selected. Glycerol solutions of both polysaccharides and their mixtures, as well as a DexMA network (obtained by crosslinking in glycerol and in the presence of a photoinitiator) were rheologically characterized to assess the role of the solvent on the chain conformation and on the mechanical properties of the networks. Interestingly, glycerol was found to deeply influence the mechanical, morphological and optical properties of the formed hydrogels, as well as to improve the crosslinking kinetic of DexMA. Furthermore, glycerol leads to more homogeneous mixtures of the two polysaccharides than those observed in aqueous buffers. As such, novel gly-semi-IPNs, with enhanced formulation and mechanical properties were prepared and characterized.
\end{abstract}

Keyworks: glycerol, polysaccharides, green solvent, semi-interpenetrating polymer networks, gellan gum, dextran methacrylate.

\section{Introduction}

Glycerol is a low-toxic, biocompatible and biodegradable polyalcohol with a wide range of applications in cosmetics and pharmaceutics ${ }^{1}$. Despite its large use in these fields, there is an active and ever increasing interest about glycerol as a by-product of the biodiesel industry ${ }^{2}$. Glycerol has been defined as "organic water" by Jérôme and co-workers ${ }^{3}$, thanks to its water typical properties, such as abundance, low-cost, high polarity, immiscibility with hydrocarbons and 
capability to dissolve several organic and inorganic compounds ${ }^{4}$. Moreover, compared to water, glycerol shows further advantages, including a higher boiling point, a lower vapour pressure and, as already pointed out, the ability to dissolve numerous organic compounds which are not soluble in water. Taking into account these advantages, glycerol has been investigated as solubilizer of a wide range of molecules in organic syntheses and extraction processes, as cosolvent in deep eutectic solvents ${ }^{5}$ (ionic liquids composed of Lewis or Bronsted acids that are able to give an eutectic mixture with a melting point much lower than either individual components, leading to a liquid system at room temperature) and, finally, as a chain transfer agent or a crosslinking agent in free radical polymerization ${ }^{6,7,8}$ or coinitiator in radical photo-polymerization. Despite its favourable properties, glycerol has been only seldom used as a solvent for polymers. Several researches dealing with polymers (especially polysaccharides) and glycerol are focused on the use of this polyol as plasticizers in hydrocolloid based films ${ }^{9-11}$, dressings for the treatment of wounds ${ }^{12}$ and humectants in skin formulations ${ }^{13}$, in concentrations up to $80 \% \mathrm{w} / \mathrm{V}$. In fact, the addition of glycerol to polymer solutions can affect some gel properties, like transparency and temperature sensitivity ${ }^{14,15}$.

The solubilization of macromolecules, especially those with a particularly high molecular weight, is a more complex process compared to the solubilization of low molecular weight compounds ${ }^{16}$. As some polymers are not soluble in water, others are insoluble in organic solvents or even in neither and considering that organic solvents are usually toxic and not biocompatible, their use is limited in several applications (e.g. food, drug delivery and tissue engineering). Therefore, many efforts have been done to find alternative and more biocompatible solvents for polymers. In this sense, ionic liquids have gained great attention thanks to their higher biocompatibility ${ }^{2,17}$; in addition, the macromolecules solubilized in such solvents show interesting and innovative structural and functional properties, different from those exhibited in conventional solvents. However, despite their advantages, there are some shortcomings of these green solvents that limit their use, mainly the high viscosity. Compared to these solvents, glycerol has a lower viscosity that makes it a very promising and alternative green solvent. Starting from these key features, herein glycerol was exploited as a novel solvent for enhancing the formulation and properties of semiinterpenetrating polymer networks (semi-IPNs), called "gly-semi-IPNs", made of two widely studied polysaccharides, dextran, which was used after derivatization with methacrylate groups (DexMA) and gellan gum (Ge). A Semi-IPN is defined by IUPAC as "A polymer comprising one or more networks and one or more linear or branched polymer(s) characterized by the penetration on a molecular scale of at least one of the networks by at least some of the linear or branched macromolecules"18. The combination of polymers with different properties provide enhanced properties to the semi-IPNs and, hence, superior performance ${ }^{19}$. Consequently, semi-IPNs have gained increasing interest in the development of hydrogels for several biomedical applications ${ }^{20,21}$. In this contest, DexMA and Ge have been extensively used for the development of water based systems in the field of drug delivery and tissue engineering as single materials or mixed with other polymers to form IPNs and semi-IPNs ${ }^{22-25}$. Dextran (Dex) is a non-toxic, bacterial and hydrophilic polysaccharide with a backbone of $\alpha-(1 \rightarrow 6)$ linked D-glucopyranose units with a low percentage of $\alpha$ $(1 \rightarrow 2), \alpha-(1 \rightarrow 3)$ and $\alpha-(1 \rightarrow 4)$ side chains. Dex has been extensively explored in tissue engineering and biomedical applications $22,23,25$. Moreover, the hydroxyl groups of Dex can be chemically functionalized leading to polymer derivatives with tuneable properties. In detail, its functionalization with methacrylate moieties (DexMA) allows hydrogel formation by radical polymerization initiated by chemical reagents or by UV light exposure. Gellan gum (Ge) is an anionic microbial polysaccharide with a tetrasaccharide repeating unit ((1-3)- $\beta$-D-glucose, (1-4)- $\beta$-D-glucuronic acid, (1-4)- $\beta$-D-glucose, and (1-4)- $\alpha$-L-rhamnose), secreted by Sphingomonas elodea. Native Ge is partially esterified 
with acyl substituents of L-glycerate and acetate at the C-2 and C-6 positions of the (1-3)-linked D-glucose. Acetyl groups are usually removed by alkaline treatment, leading to deacetylated Ge, which is capable of forming physical cation-induced hydrogels. This property makes Ge suitable for the development of in situ gelling controlled release systems. In this work, the key role of glycerol as a green solvent, over the conventional aqueous buffer (HEPES), was investigated for the formation of DexMA/Ge based gly-semi-IPNs. The crosslinking kinetic of the resulting scaffolds was studied; rheological, dynamo-mechanical and morphological properties were elucidated. The formation of DexMA/Ge semi-IPNs in glycerol was extremely faster than that in water. Furthermore, DexMA/Ge semi-IPNs prepared in glycerol exhibited high optical transparency and homogeneity, suggesting suitable properties both for biomedical and 3D printing applications.

\section{Experimental}

\subsection{Materials}

Dextran from Leuconostoc ssp. (Dex) $\left(\mathrm{M}_{\mathrm{w}}=40 \times 10^{3}\right), 4-\mathrm{N}, \mathrm{N}-$ dymethylaminopyridine (DMAP), glycidyl methacrylate (GMA), 2-hydroxy-4'-(2-hydroxy-etoxy)-2-methyl-propriophenone (Irgacure 2959), glycerol, N-(2Hydroxyethyl)piperazine-N'-(2-ethanesulfonic acid) (HEPES), fluorescein sodium salt (FL) and dimethyl sulfoxide (DMSO) were provided by Sigma-Aldrich (Italy). Low acyl Gellan gum sodium salt (Ge) $\left(\mathrm{Mw}_{\mathrm{w}}=2.5 \times 10^{6}\right)$ was purchased from CP Kelco (USA). All chemicals were used as received without further purification.

\subsection{Methods}

\subsubsection{Synthesis of methacrylated Dextran (DexMa)}

DexMa synthesis was carried out by dissolving $10 \mathrm{~g}$ of Dex in $100 \mathrm{~mL}$ of anhydrous DMSO and $2 \mathrm{~g}$ of DMAP ${ }^{23,26,27}$. After DMAP dissolution, $1.74 \mathrm{~mL}$ of GMA were added in order to obtain a DexMA degree of substitution (DS) of $20 \%$ $\mathrm{mol} / \mathrm{mol}$ (mol of methacrylate per mol of Dex repeating units). The reaction was carried out for $48 \mathrm{~h}$ at room temperature under magnetic stirring and the $\mathrm{pH}$ was then adjusted to 7 with $\mathrm{HCl} 1 \mathrm{~N}$ to neutralize DMAP. Finally, the mixture was dialyzed until constant conductivity was reached (Visking tubes, MW cut-off: 12,000-14,000) and the samples were freeze-dried (yield 84-89\%).

\subsubsection{Sample preparation}

Samples were prepared using two different solvents: glycerol or HEPES buffer (pH 7.2-7.4, 5·10-2 M). Starting stock solutions of DexMA (5\% w/V) and Ge (at concentrations ranging from 0 to $2 \% \mathrm{w} / \mathrm{V}$ ) were prepared at $80^{\circ} \mathrm{C}$ and $100^{\circ} \mathrm{C}$ respectively. Then, $2 \mathrm{~g}$ each polymer stock solutions were mixed and magnetically stirred for 10 minutes. Finally, the photoinitiator Irgacure 2959, was added to the polymer solution (6 $\mu \mathrm{L}$ of a $16 \% \mathrm{w} / \mathrm{V}$ solution for each g of solution) and the mixture was gently stirred for 1 minute. The solution was irradiated using a mercury UV lamp ( $\lambda$ maximum $365 \mathrm{~nm}$, light intensity $20 \mathrm{~mW} / \mathrm{cm}^{2}$ ), leading to the semi-IPN formation (Fig.8).

\subsubsection{Rheological characterization}

Rheological experiments were carried out using a stress-controlled Discovery Hybrid Rheometer-1 (TA instruments New Castle, DE, USA), equipped with a Peltier temperature controlling unit. Frequency sweep experiments were performed at $25^{\circ} \mathrm{C}$ in the range of $0.01-100 \mathrm{~Hz}$, applying a constant deformation in the linear regime $(\gamma=1 \%)$ by using a grained parallel plate geometry (diameter $=20 \mathrm{~mm}$ ). 
Flow curves of the solutions were performed using a cone-plate geometry (diameter $=40 \mathrm{~mm}$, cone $1^{\circ}$, gap $=0.027$ $\mathrm{mm}$ ) in the range of $0.001-1000 \mathrm{~s}^{-1}$. All the measurements were done at $25^{\circ} \mathrm{C}$.

\subsubsection{Fluorescence assay}

To assess the role of glycerol as chain transfer agent, an automated fluorescence assay was carried out on a Wallac 1420 VICTOR2 multilabel counter (Perkin-Elmer, USA) at the excitation wavelength of $485 \mathrm{~nm}$ and emission wavelength of $535 \mathrm{~nm}$. The measurements were done in plates with 96 white flat-bottom wells. A stock solution of FL was prepared by weighing $44 \mathrm{mg}$ of $\mathrm{FL}$, dissolving it in $100 \mathrm{ml}$ of phosphate buffer (PBS) (75 mM, pH 7.0), and then storing it in the dark at $4^{\circ} \mathrm{C}$. The working solutions of FL (78 $\mathrm{nM}$ ) in both solvents (HEPES or glycerol) were prepared daily, diluting $0.167 \mathrm{ml}$ of the stock solution in $25 \mathrm{ml}$ of HEPES or glycerol. To perform the test, $95 \mu \mathrm{l}$ of these two working solutions were placed in the well plate, and then $5 \mu \mathrm{l}$ of a solution of the photoinitiator I-2959 were added to reach the final concentration of $1 \% \mathrm{w} / \mathrm{V}$ in each well.

\subsubsection{Variable Pressure Scanning Electron Microscopy}

The hydrogel morphology was studied using a variable pressure scanning electron microscope (VP-SEM, Hitachi SU3500). Cross-sectioned hydrated samples were observed without conductive coating. VP-SEM combined with Peltier coolstage control allowed high-resolution imaging of hydrated specimens under varying pressure conditions as well as limiting water vapour loss through the control of stage temperature. A thin film of distilled water was deposited onto the hydrated samples at room temperature, in order to limit the water-loss during low vacuum procedures. By controlling the chamber pressure and the cooling temperature, the collapse of the surface pores was avoided. All the samples were observed at an accelerating voltage of $8 \mathrm{KV}{ }^{28}$, considering a working distance between 5 and $6 \mathrm{~mm}$ and a correlative pressure/temperature stabilized at $60 \mathrm{~Pa} /-25{ }^{\circ} \mathrm{C}$ before the measurements. The pore size and area of hydrogels were quantified using Fiji software (NHI-USA).

\subsubsection{Dynamo-mechanical characterization}

For the mechanical characterization of the hydrogels, a software-controlled dynamometer, TA-XT2i Texture Analyzer (Stable Micro Systems, UK) with a $5 \mathrm{~kg}$ load cell, was used. Compression tests were performed by recording the resistance of cylindrical samples (diameter $22 \mathrm{~mm}$, height $30 \mathrm{~mm}$ ) to the compression of an ebonite cylinder probe with a diameter of $10 \mathrm{~mm}$. The pre-test, test and post-test speeds were $2.0 \mathrm{~mm} / \mathrm{s}, 1.0 \mathrm{~mm} / \mathrm{s}, 1.0 \mathrm{~mm} / \mathrm{s}$ respectively with an acquisition rate of 10 points/s. The end point of the test was monitored and referred to as ultimate compressive stress, $\sigma$, and ultimate compressive deformation, $\gamma$, respectively. The Young modulus (E) was calculated as the slope of the stress-versus strain curve, in the range of strain from 0 to $10 \%$. The experiments were carried out at $25^{\circ} \mathrm{C}$ and in triplicate.

\section{Results and discussion}

\subsection{Characterization of polymer solutions}

Viscosity is considered one of the most peculiar and important features of glycerol, which makes this solvent a thickener or body agent suitable for food and pharmaceutical applications. In fact, glycerol shows the viscosity value of $954 \mathrm{mPa} \cdot \mathrm{s}$ at $25^{\circ} \mathrm{C}$, which is three order of magnitude higher than that of the water ${ }^{1}$ and lower than that of emerging solvents (i.e. deep eutectic solvents or ionic liquid widely used for i.e. polymer synthesis and/or 
solubilisation). A further reduction of the glycerol viscosity can be obtained by increasing the temperature, thus allowing the solubilization of numerous compounds including several high molecular weight polymers. Therefore, glycerol was selected as a green solvent for enhancing the formulation of semi-IPNs materials useful for biomedical applications.

Table 1 shows the concentration and temperature values at which certain polymers form completely clear solutions in glycerol.

Table 1 Concentration $(\% \mathrm{w} / \mathrm{w})$ and temperature $\left({ }^{\circ} \mathrm{C}\right)$ values at which certain polymers form completely clear solutions in glycerol.

\begin{tabular}{ccc}
\hline Polymer & Concentration $(\%$ & Temperature $\left({ }^{\circ} \mathrm{C}\right)$ \\
& w/w) & \\
\hline Gelatin A & Up to 10 & 100 \\
Dextran & Up to 3 & 80 \\
DexMA & Up to 10 & 80 \\
Hyaluronan tetrabutylammonium salt & Up to 10 & 80 \\
Pluronic F127 & Up to 1.5 & 80 \\
\hline
\end{tabular}

Among the tested polymers (data not shown), the DexMA and Ge glycerol solutions showed viscosity values suitable for the preparation of semi-IPNs with a higher homogeneity and enhanced properties compared to the same systems prepared in water and/or aqueous buffers. In fact, it is well known that solvents can have a great impact on the polymer conformation, enabling the dissolution or the phase separation ${ }^{29}$. For this reason, the effect of glycerol on the conformation of DexMA and Ge was investigated by rheological measurements and compared to the behaviour exhibited by the same polymers in aqueous buffer. Fig. 1a and 1b show the complex viscosity $\left(\eta^{*}\right)$ of DexMA and Ge solutions as a function of the polymer concentration at $25^{\circ} \mathrm{C}$.

(a)

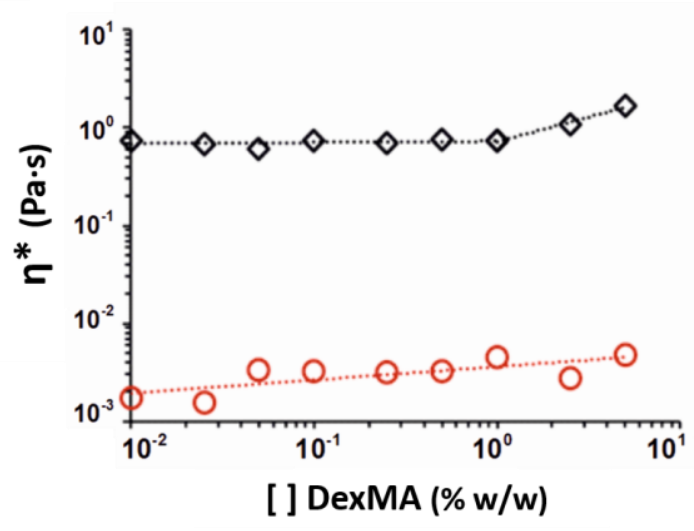

(b)

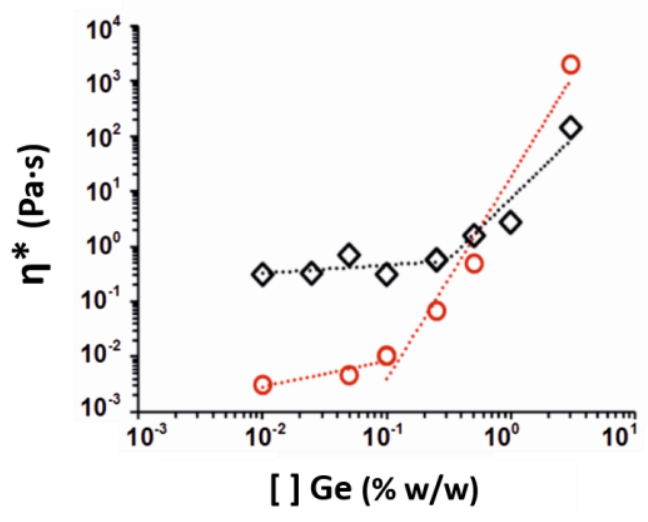

Fig. 1 Complex viscosity $\left(\eta^{*}\right)$ at $1 \mathrm{~Hz}$ as a function of the polymer concentration for DexMA (a) and Ge (b) in HEPES (O) or glycerol $(\diamond)$ at $25^{\circ} \mathrm{C}$. Dashed lines are only an eye-guide. 
Complex viscosity of DexMA (Fig. 1a) in HEPES was slightly dependent on concentration in the whole investigated range- whereas, in glycerol, the $\eta^{*}$ of DexMA was slightly dependent on concentrations up to $1 \%$ w/w, while for higher concentrations $\eta^{*}$ values increased. One of the factors determining the magnitude of the viscosity and, hence, the magnitude of $\eta^{*}$, beside the solvent viscosity, is the hydrodynamic volume of DexMA molecules and, hence, the different expansion of the coil in the two solvents. Therefore, these results suggest that DexMA macromolecules are characterized by a more expanded coil in glycerol compared to HEPES, with corresponding significantly higher $\eta^{*}$ values. This can be explained in terms of more favourable interactions among the polymer chains and glycerol compared to those established with water, due to the hydrophobic character of methacrylate moieties present along the polysaccharide macromolecules. A more remarkable difference in terms of complex viscosity behaviour was observed for Ge solutions, whose curves as a function of the polymer concentrations in HEPES and glycerol are reported in Fig. 1b. Specifically, in the case of Ge, a critical concentration was observed both in HEPES and in glycerol. In the semi-diluted domain - below the "critical concentration", $c^{*}$, that marks the onset of a significant coil overlap and interpenetration, Ge solutions behaved like "free" random coil polymers in glycerol. Above the c*, a very strong concentration dependence of $\eta^{*}$ was observed. The higher slope for Ge in HEPES compared to glycerol could be ascribed to a stiffer conformation adopted by the polymer chains in this solvent due to their double-stranded conformation. These results suggest that from the thermodynamic point of view HEPES behaves as a better solvent for Ge compared to glycerol.

In order to investigate the conformation of Ge chains in both solvents, viscosity curves of Ge solutions in HEPES or in glycerol were recorded at concentrations ranging from 0.25 to $2 \%$ (Fig. 2).

(a)

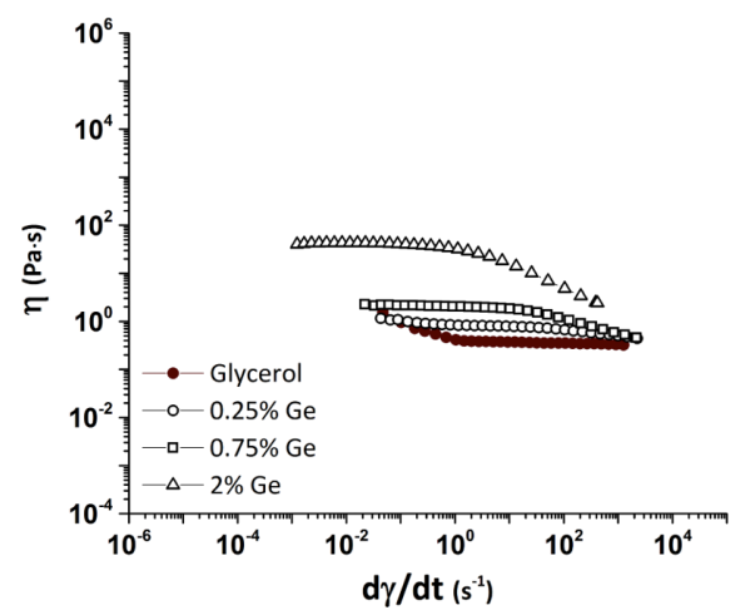

(b)

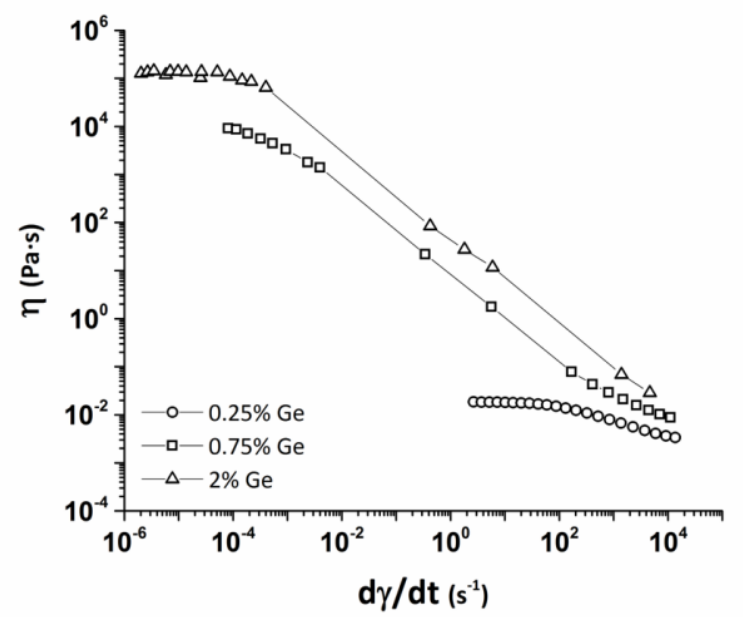

Fig. 2 Viscosity curves (at $25^{\circ} \mathrm{C}$ ) of Ge solutions $(0.25 \%, 0.75 \%, 2 \%, w / w)$ in (a) glycerol and (b) HEPES. In Fig. 2 (a), the flow curve of glycerol (full symbols) is also reported.

The $0.25 \%$ Ge solution in HEPES (Fig. 2 b) show a behaviour close to a Newtonian flow, whilst more concentrated Ge solutions $(0.75 \%$ and $2 \% \mathrm{w} / \mathrm{w})$ exhibited a wide shear thinning behaviour. This flow behaviour could be explained taking into account also the stiffness of the Ge chains in aqueous media due to the formation of double-stranded helices $^{30,31}$. On the other hand, Ge solutions in glycerol were characterized by an extended quasi-Newtonian plateau up to $2 \%$ (Fig. 2a); therefore, the Ge chains, assuming coiled shapes in glycerol, are more flexible. Glycerol shows a 
lower dielectric constant compared to that of water, 42.6 and 80 respectively, that is responsible of the reduction of the electrostatic repulsion among the polymer chains leading to the reduction of their stiffness. Moreover, glycerol could form strong hydrogen bonds with Ge chains thanks to its $\mathrm{OH}$ groups, resulting in a further reduction of the intermolecular interactions among Ge chains ${ }^{11}$.

Taking advantage of the different conformation that Ge chains assumes in glycerol, it is possible to obtain homogenous Ge solutions at higher polymer concentrations compared to those in HEPES. In this contest, the significant viscosity reduction of Ge solutions in glycerol was here exploited with the aim to prepare homogenous mixtures of Ge and DexMA useful for DexMa/Ge gly-semi-IPNs development.

\subsection{Kinetic of DexMA crosslinking process}

Rheological characterization of the polymer solutions showed that glycerol can act as a promising and innovative solvent for both DexMA and Ge, leading to solutions with different and enhanced rheological properties in terms of viscosity. DexMA is a photocrosslinkable polysaccharide derivative able to form gels when its aqueous solutions are crosslinked by UV light exposure in the presence of I-2959 as photoinitiator. To investigate the effect of solvent on DexMA crosslinking process, solutions of $5 \% \mathrm{w} / \mathrm{w}$ DexMA in glycerol were exposed to UV curing for different times in the presence of I-2959 and the obtained networks were analyzed and compared to those formed by using HEPES as a solvent. Fig. 3a shows the $\mathrm{G}^{\prime}$ values of $5 \% \mathrm{w} / \mathrm{w}$ DexMA, recorded at $1 \mathrm{~Hz}$ in both solvents, plotted as a function of the crosslinking time. Upon UV irradiation, the storage moduli G' rapidly increased and reached a plateau within 1 minute when glycerol was used, meaning that a very fast crosslinking reaction occurred. The polymerization of the methacrylate groups on DexMA chains in glycerol was confirmed by FT-IR analyses (Fig. 3b), where a reduction in the IR peak intensity in the double bond region at $1640 \mathrm{~cm}^{-1}(\mathrm{C}=\mathrm{C})$ was observed. On the other hand, when an aqueous HEPES solution was used, the kinetic was slower, showing a complete polymerization after 15 minutes of UVexposure. Furthermore, the storage moduli of the networks were higher in glycerol ( $\mathrm{G}^{\prime} \sim 5 \mathrm{kPa}$ ) compared to the $\mathrm{G}^{\prime}$ values in HEPES $\left(\mathrm{G}^{\prime} \sim 1 \mathrm{kPa}\right)$ suggesting that glycerol led to an enhancement in the mechanical properties of the DexMA networks and to a remarkable reduction of the crosslinking time. Crosslinking reactions were also carried out on DexMA 5\% w/w solutions at different glycerol and HEPES ratios (Fig. 3c), glycerol:HEPES, 25:75, 50:50, 75:25.

A strong dependence of the time required for the complete formation of the DexMa network (Fig. 3d) was observed and the $G^{\prime}$ values increased from HEPES to glycerol, thus suggesting the enhancing role of glycerol in the radical reaction and in the network architecture, as well. The possible role of glycerol to act as a photoinitiator was investigated by exposing solutions of 5\% w/w DexMA in glycerol to UV lights for 3 hours, without adding I-2959 to the solutions. In this case, no crosslinking of methacrylate moieties was observed, confirming the ability of glycerol to act only as co-initiator, being not able to start the polymerization process. 
(a)

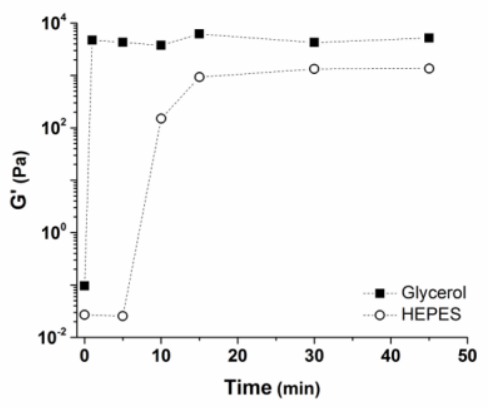

(d)

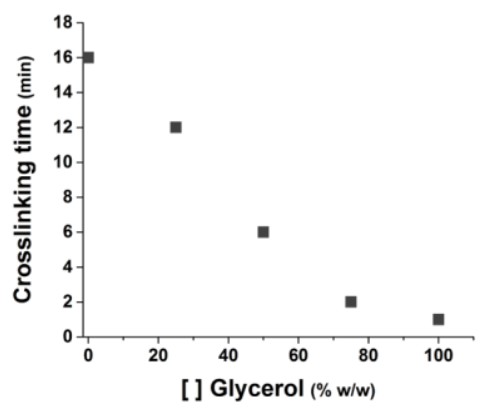

(b)

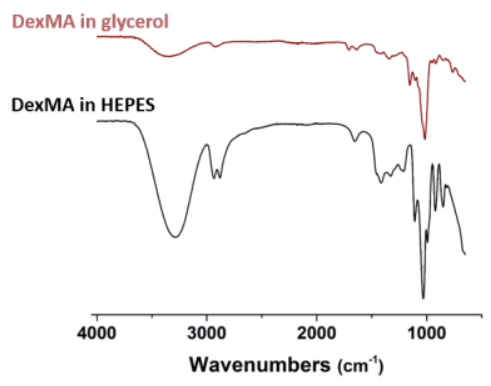

(e)

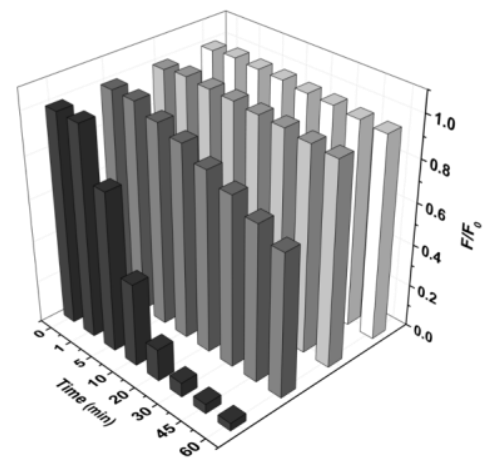

(c)

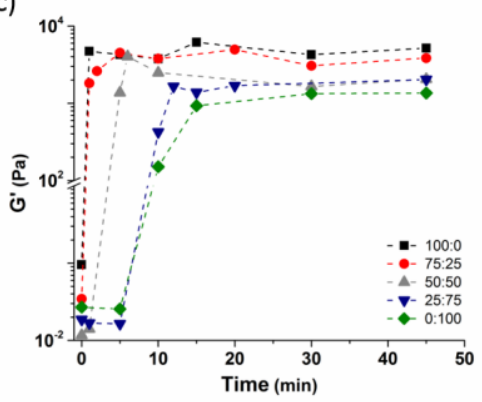

Fig. 3 (a) Storage modulus, G', at $1 \mathrm{~Hz}$, as a function of time for 5\% w/w DexMA blend prepared in pure glycerol or HEPES. (b) IR spectra of DexMA and 5\% DexMA gel in glycerol after 1 minute of UV-exposure. (c) Storage modulus, G', at $1 \mathrm{~Hz}$, as a function of time for $5 \% \mathrm{w} / \mathrm{w}$ DexMA blend prepared in the presence of different glycerol:water ratios $(100: 0,75: 25,50: 50,25: 75$ and 0:100). (d) Crosslinking reaction time of DexMA as a function of the glycerol concentration. (e) Consumption profiles of fluorescein (FL) in glycerol and HEPES with or without I-2959 after UV exposure.

It is known that glycerol can act as a multifunctional chain transfer agent, allowing crosslinking reactions at high concentrations $^{6}$. To assess the role of glycerol as chain transfer agent in the crosslinking process of DexMA, a fluorescence assay was performed. Fluorescein (FL) was used as a fluorescent probe thanks to its high sensitivity to radicals and solubility in both water and glycerol. To determine the contribution of glycerol to radical transfer, the fluorescence intensity of FL was studied in glycerol with or without the photoinitiator I-2959 after exposure of the solutions to the UV-light (Fig. 3e). For an appropriate comparison, the fluorescence intensity of FL in HEPES with or without I-2959 was also investigated. In HEPES, even after $60 \mathrm{~min}$ of UV-exposure in the presence of the photoinitiator, no reduction of fluorescence was observed, suggesting that the radicals generated by the photoactivation of I-2959 are not enough to significantly reduce the fluorescence of FL and they are also not able to easily propagate in an aqueous environment. On the other hand, when FL was used in glycerol without the addition of I2959, a gradual decrease of fluorescence was observed. However, a more remarkable reduction of fluorescence was observed when the photoinitiator was added to glycerol-FL mixture leading to a total consumption of the fluorescent probe after $50 \mathrm{~min}$ of UV-exposure. These results confirmed that the glycerol acts as radical chain transfer, when radicals are previously formed, according to the paper of Osmanov and colleagues ${ }^{7}$.

Importantly, the effects of glycerol in the DexMA crosslinking reaction allows a significant reduction of the amount of I-2959 required for a fast network formation (Table $2 \mathrm{a}$ and $2 \mathrm{~b}$ ). Fixing 20 minute of UV-exposure time, the amount of I-2959 required to form a complete DexMA network is 400 -fold lower in glycerol compared to HEPES. At the same 
time, fixing the amount of 12950 at $0.0025 \%$, a complete network formation in 1 min is obtained in glycerol, whereas in HEPES no wall-to-wall network formation is evident until $1 \mathrm{~h}$. Both, the reduction of the photo-initiator concentration and the faster kinetic of the crosslinking reaction, represent a significant advantage in the development of engineered scaffolds.

Table 2 Physical state, according to the rheological data, of 5\% DexMa samples, as a function of the UV-exposure time, in the presence of several I-2959 concentrations, prepared in HEPES (a) or glycerol (b). "L" corresponds to liquid, whilst "S" to the fully cross-linked network

(a)

\begin{tabular}{ccccccccc}
\hline Amount of I-2959 (\%) & \multicolumn{7}{c}{ Time $(\min )$} & \multicolumn{1}{c}{} \\
\hline 0 & 1 & 5 & 10 & 15 & 20 & 30 & 45 & 55 \\
\hline 0.0025 & $\mathrm{~L}$ & $\mathrm{~L}$ & $\mathrm{~L}$ & $\mathrm{~L}$ & $\mathrm{~L}$ & $\mathrm{~L}$ & $\mathrm{~L}$ & $\mathrm{~L}$ \\
0.0025 & $\mathrm{~L}$ & $\mathrm{~L}$ & $\mathrm{~L}$ & $\mathrm{~L}$ & $\mathrm{~L}$ & $\mathrm{~L}$ & $\mathrm{~L}$ & $\mathrm{~L}$ \\
0.025 & $\mathrm{~L}$ & $\mathrm{~L}$ & $\mathrm{~L}$ & $\mathrm{~L}$ & $\mathrm{~L}$ & $\mathrm{~L}$ & $\mathrm{~L}$ & $\mathrm{~L}$ \\
0.5 & $\mathrm{~L}$ & $\mathrm{~L}$ & $\mathrm{~L}$ & $\mathrm{~L}$ & $\mathrm{~L}$ & $\mathrm{~L} / \mathrm{S}$ & $\mathrm{L} / \mathrm{S}$ & $\mathrm{S}$ \\
0.1 & $\mathrm{~L}$ & $\mathrm{~L}$ & $\mathrm{~L}$ & $\mathrm{~L} / \mathrm{S}$ & $\mathrm{L} / \mathrm{S}$ & $\mathrm{S}$ & $\mathrm{S}$ & $\mathrm{S}$ \\
0.2 & $\mathrm{~L}$ & $\mathrm{~L}$ & $\mathrm{~L} / \mathrm{S}$ & $\mathrm{L} / \mathrm{S}$ & $\mathrm{S}$ & $\mathrm{S}$ & $\mathrm{S}$ & $\mathrm{S}$ \\
\hline
\end{tabular}

(b)

\begin{tabular}{ccccccccc}
\hline Amount of I-2959 (\%) & \multicolumn{9}{c}{ Time (min) } & \multicolumn{1}{c}{} \\
& 1 & 5 & 10 & 15 & 20 & 30 & 45 & 55 \\
\hline 0 & $\mathrm{~L}$ & $\mathrm{~L}$ & $\mathrm{~L}$ & $\mathrm{~L}$ & $\mathrm{~L}$ & $\mathrm{~L}$ & $\mathrm{~L}$ & $\mathrm{~L}$ \\
0.00025 & $\mathrm{~L}$ & $\mathrm{~L}$ & $\mathrm{~L}$ & $\mathrm{~L} / \mathrm{S}$ & $\mathrm{S}$ & $\mathrm{S}$ & $\mathrm{S}$ & $\mathrm{S}$ \\
0.0025 & $\mathrm{~S}$ & $\mathrm{~S}$ & $\mathrm{~S}$ & $\mathrm{~S}$ & $\mathrm{~S}$ & $\mathrm{~S}$ & $\mathrm{~S}$ & $\mathrm{~S}$ \\
0.025 & $\mathrm{~S}$ & $\mathrm{~S}$ & $\mathrm{~S}$ & $\mathrm{~S}$ & $\mathrm{~S}$ & $\mathrm{~S}$ & $\mathrm{~S}$ & $\mathrm{~S}$ \\
0.5 & $\mathrm{~S}$ & $\mathrm{~S}$ & $\mathrm{~S}$ & $\mathrm{~S}$ & $\mathrm{~S}$ & $\mathrm{~S}$ & $\mathrm{~S}$ & $\mathrm{~S}$ \\
0.1 & $\mathrm{~S}$ & $\mathrm{~S}$ & $\mathrm{~S}$ & $\mathrm{~S}$ & $\mathrm{~S}$ & $\mathrm{~S}$ & $\mathrm{~S}$ & $\mathrm{~S}$ \\
0.2 & $\mathrm{~S}$ & $\mathrm{~S}$ & $\mathrm{~S}$ & $\mathrm{~S}$ & $\mathrm{~S}$ & $\mathrm{~S}$ & $\mathrm{~S}$ & $\mathrm{~S}$ \\
\hline
\end{tabular}

\subsection{Morphology of DexMA networks in glycerol}

Glycerol deeply affected not only the kinetic of the DexMA crosslinking reaction, but also the visual appearance of the photo cross-linked network (Fig. 4a). Networks prepared in pure glycerol were optically transparent. When HEPES was added to glycerol (glycerol:HEPES ratio of 75:25), the networks became less transparent, while by increasing HEPES amounts (glycerol:HEPES ratio of 50:50, 25:75), DexMA systems were opaquer and completely white in the presence of pure HEPES, according to previous literature data ${ }^{32}$. It is well known that glycerol is widely employed to obtain tissues with optical transparency thanks to its ability to reduce tissue light scattering. Moreover, glycerol can also be responsible for the conformational changes of polymer chains, enhancing the anisotropy of the nanostructures, thus leading to reduced crystallinity and scattering of some polymeric scaffolds ${ }^{33}$. For this reason, to assess the possibility of conformational changes of polymer chains induced by the solvent, the cross-sectional microstructures of gels were examined by variable pressure scanning electron microscopy (VP-SEM) (Fig. 4b). 
a)

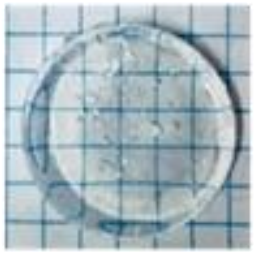

100:0

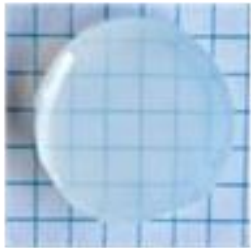

$75: 25$

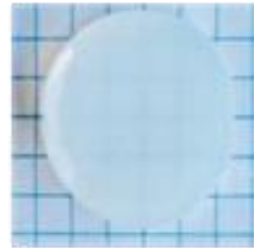

$50: 50$

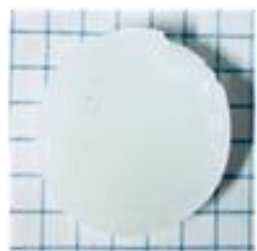

$0: 100$

b)
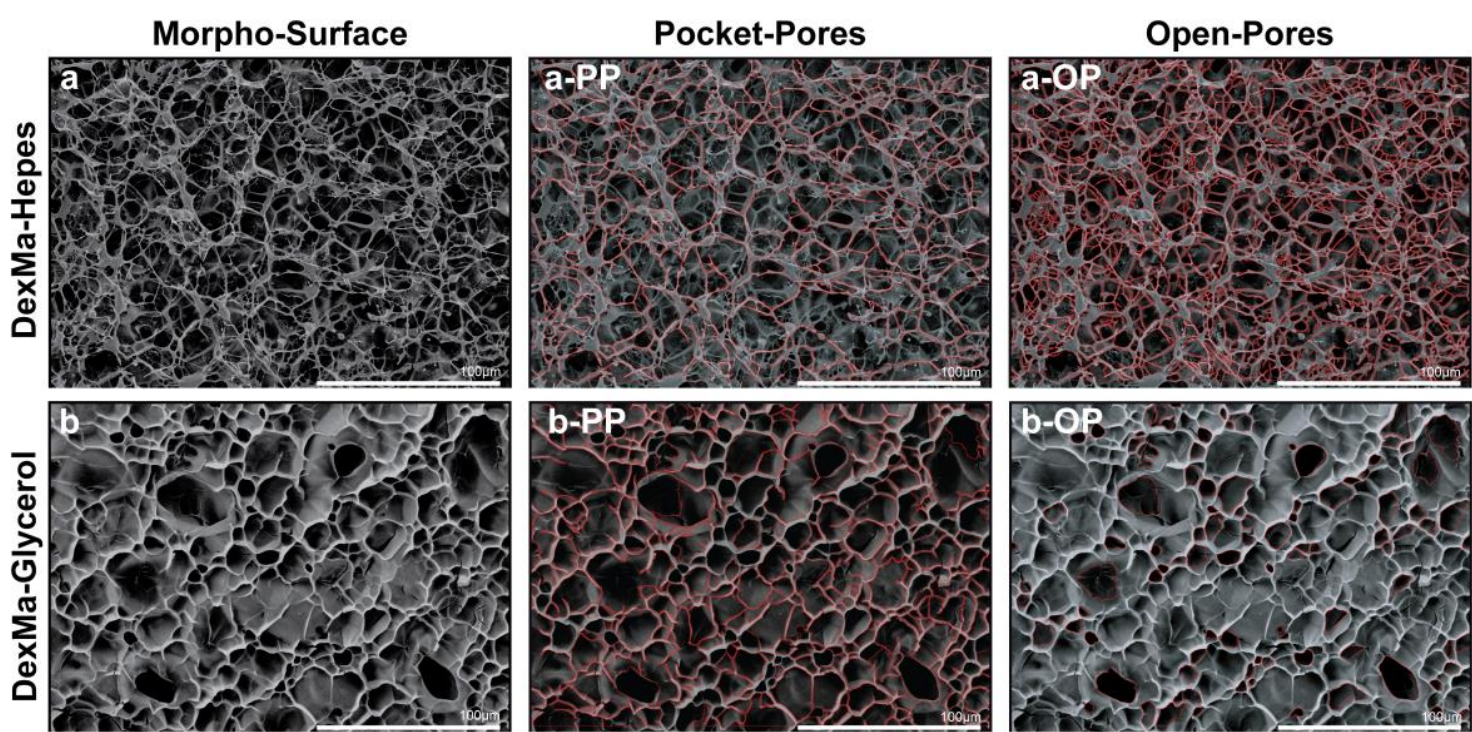

Porous Analyses
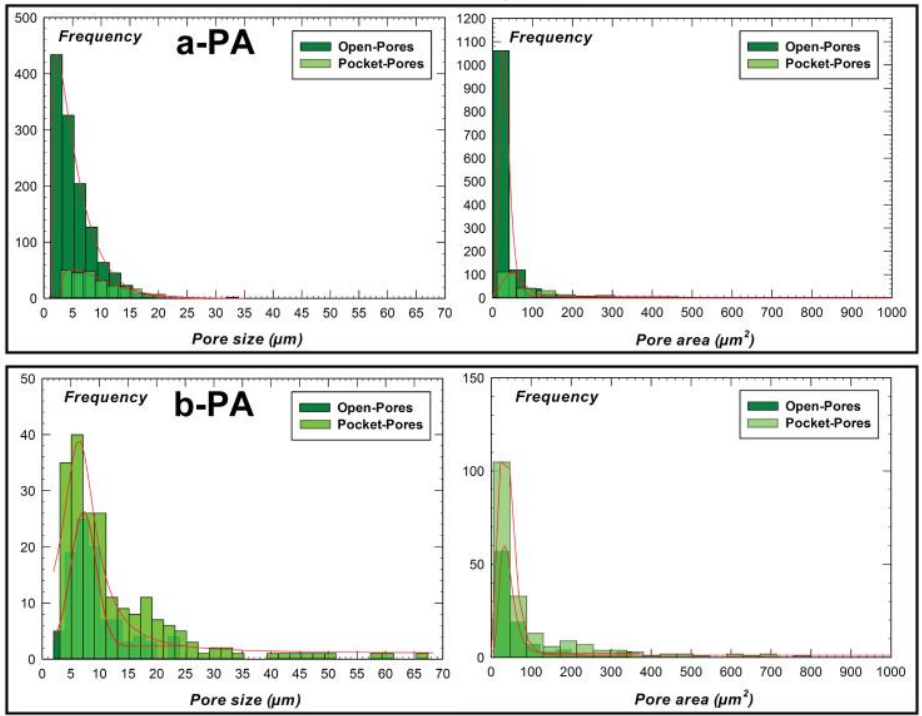

Fig.4 (a) Pictures of 5\% w/w DexMA samples in glycerol (100:0), in mixture of glycerol and HEPES at different ratios (75:25 and 50:50) or only HEPES (0:100). (b) VP-SEM micrographs (left): 5\% DexMA scaffold in HEPES or glycerol, 2D contour maps in red lines of the pocket and open pores network of DexMA-HEPES (a-PP and a-OP) and DexMAglycerol (b-PP and b-OP). Histograms (right) show the frequency distributions of the pores dimension and surface areas of DexMa-HEPES (a-PA) and DexMA-glycerol gels (b-PA).

The low vacuum SEM technique was able to evidence the formation of the heterogeneous porosity of the hydrogets in their native state. By applying the surface morphology analysis, the pore size, pore area, and their correlated 
distributions were quantitatively determined (Fig. 4b). The cross-sectional microstructures of the DexMA networks in HEPES exhibit a delicate and fragile multiscale porous structures with interconnected pockets and open pores, as shown in Fig. 4b: a. Quantitative morphometric measurements of both pockets and open pores inside the polymeric hydrogels were investigated on a probe area of $252 \mu \mathrm{m}$ by $189 \mu \mathrm{m}^{34,35}$. A 2D contour maps of 250 pocket pores and 1242 open pores distribution with evident formation of sub-porosity were measured, as shown in Fig. 4b: a-PP and 4b: a-OP and in Fig. S1 SI:a. By plotting the morphometric data of open pores $\left(2-18 \mu \mathrm{m}, 20-180 \mu \mathrm{m}^{2}\right)$ that were slightly smaller in dimensions and surface area than the pocket ones (4-21 $\left.\mu \mathrm{m}, 35-500 \mu \mathrm{m}^{2}\right)$, we were able to establish that the amount of open pores is two orders of magnitude higher than that of the pockets pores. The frequency profiles of the histograms exhibit a distribution centred around the mean values of the pocket pore size of $4.99 \pm 0.26 \mu \mathrm{m}(44.30$ $\pm 2.45 \mu \mathrm{m}^{2}$, projected area) and of the open pore size of the $2.22 \pm 0.23 \mu \mathrm{m}\left(25.57 \pm 4.73 \mu \mathrm{m}^{2}\right.$, projected area; Fig. $5 \mathrm{a}-$ PA). Morphological surface network changes occurred in the swollen DexMA hydrogels by addition of glycerol, including alteration of size distribution of the pores and an apparent decrease of porous microstructure and its interconnection features (see in Fig. 4b: b). The pores exhibit densely packed pore walls, which have an impact on the local loading capacity of the molecules (i.e. drugs, proteins and so on) or cells, considering the potential biomedical applications of these scaffolds, and on their mechanical behaviour. Interestingly, glycerol significantly affected the morphological porosity, changing the open pores (97 pores) in pockets pores (202 pores) with a relevant increase in the size and area of the blind pores (Fig. 4b: b and Fig. S1 SI: b). The frequency distributions of pocket pores (4-65 $\mu \mathrm{m}$, 26-700 $\left.\mu \mathrm{m}^{2}\right)$ increase their morphometric properties compared to that of the open pores $\left(3-23 \mu \mathrm{m}, 27-330 \mu \mathrm{m}^{2}\right)$. The mean values of the pocket pore size of $6.43 \pm 0.33 \mu \mathrm{m}\left(32.85 \pm 1.57 \mu \mathrm{m}^{2}\right.$, projected area) became similar to that of the open pore of $7.20 \pm 0.22 \mu \mathrm{m}\left(33.10 \pm 5.78 \mu \mathrm{m}^{2}\right.$, projected area) with a relevant increase in the number weighted frequency distribution (or bin counts) of the blind pores. An increase of a thick and dense layer of the polymeric wall could be ascribed to the different kinetic of the cross-linked process: in glycerol the cross-linking of DexMA is very fast, thus the polymer chains are immediately locked in the conformation assumed in the solvent. On the other side, in HEPES, the kinetic is slower and the polysaccharide chains can rearrange while the polymerization proceeds. The presence of glycerol induced an increase of the wall thickness within a smooth surface, which indicates the formation of a compact network structure.

\subsection{DexMA/Ge gly-semi-IPNs}

Taking into account the interesting results obtained using DexMA and Ge in glycerol, mixtures of the two polymers were tested in the same solvent. For this purpose, mixtures of DexMA and Ge were prepared and characterized in terms of rheology and compared to the solutions prepared in HEPES; then, semi-IPNs were prepared by exposing their solutions to UV light (Fig. 5) and the obtained networks were characterized in terms of kinetic of the crosslinking process, rheological and dynamo-mechanical properties. 
a)

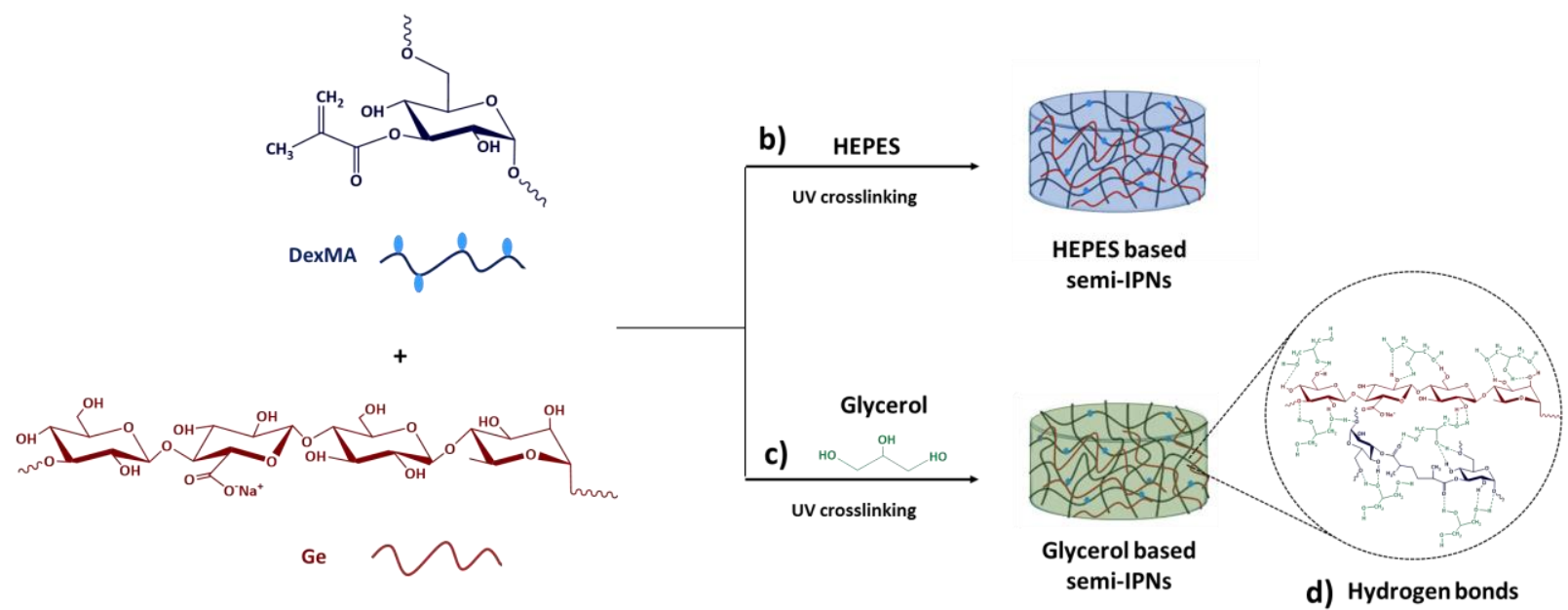

Fig 5: Scheme of DexMA/Ge semi-interpenetrating polymer network formation. a) Structures and schematic representation of DexMA and Ge. b) Formation of DexMA/Ge semi-IPNs in pure HEPES after UV curing. c) Formation of gly-semi-IPNs in pure glycerol after UV curing. d) Schematic representation of the possible interactions via hydrogen bonds among glycerol, DexMA and Ge in gly semi-IPNs.

To test the interactions between DexMA and Ge in glycerol solutions in the experimental conditions adopted for gel formation, mixtures of $5 \% \mathrm{w} / \mathrm{w}$ DexMA and different concentrations of Ge (ranging from $0.25 \%$ to $2 \%$ w/w) were characterized in both steady shear and in oscillatory regime using rheology and compared to those prepared in HEPES. Flow curves reported in Fig. 6 show that DexMA exhibited a Newtonian behaviour within the investigated shear rate range in both glycerol and HEPES, while viscosity curves of the DexMA/Ge mixtures were deeply different in the two solvents. The different rheological behaviour of the mixtures is in agreement with the above reported results (Fig. 1a, $2 \mathrm{a}$ and $2 \mathrm{~b}$ ) and could be ascribed to the different rheological behaviour of Ge in the two solvent and particularly to its ability to form very stiff gels in aqueous environment even at low concentration. As a consequence, mixtures exhibited a Newtonian behaviour at very low shear rate and a shear thinning behaviour at high shear rates in HEPES; while in glycerol all the mixtures showed a quasi-Newtonian behaviour in the whole range of the investigated shear rate. Moreover, in that range of shear the mixture viscosities increased as the Ge concentration increased. Therefore, the results in Fig. 6 show that it is possible to tune the viscosity of the blend by varying the Ge concentration. Comparing the flow curves of DexMA/Ge blends in both solvents, it is evident that the behaviour of the blend solutions is dominated by Ge both in HEPES and glycerol. However, the effect of Ge concentration is more remarkable in HEPES than in glycerol, due to the ability of Ge to adopt a double-stranded conformation in HEPES. 
(a)

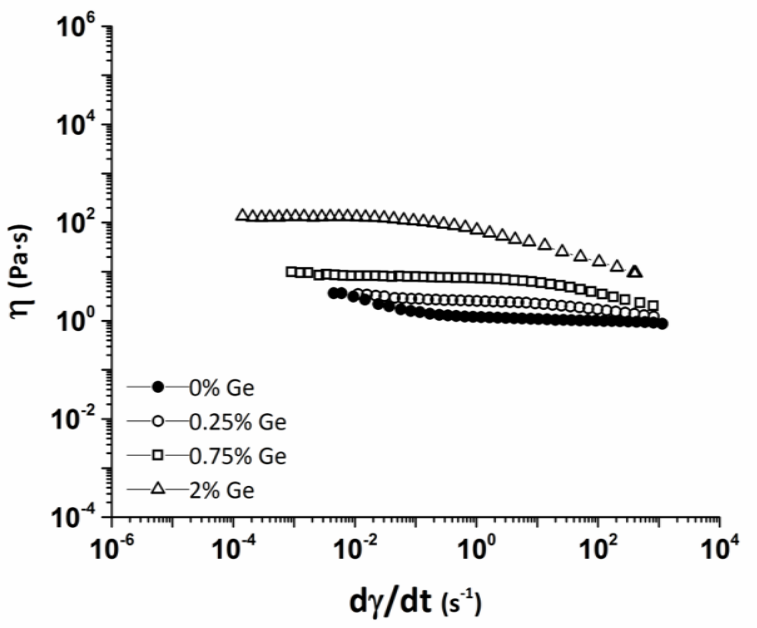

(b)

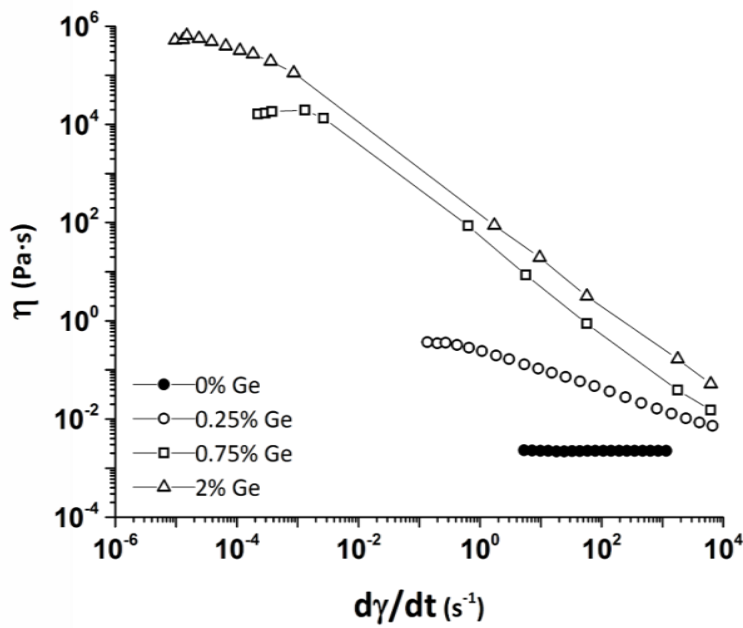

Fig. 6 Viscosity curves of 5\% DexMA solutions and mixtures of $5 \%$ w/w DexMA with $0.25 \%, 0.75 \%$ and $2 \%$ w/w Ge in glycerol (a) and HEPES (b). 
(a)

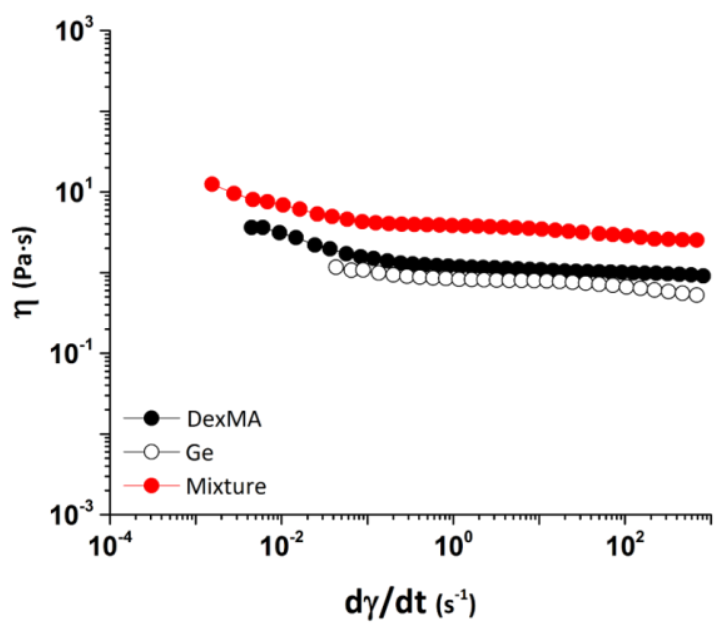

(b)

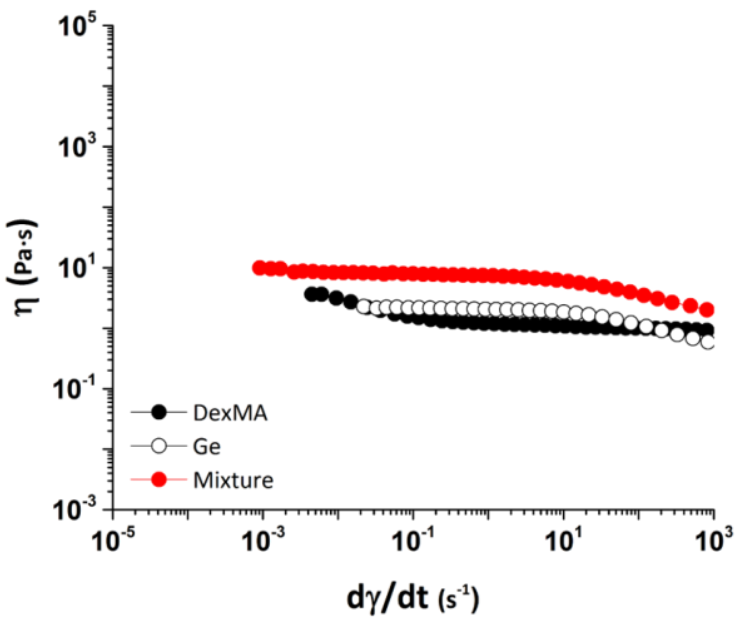

(c)

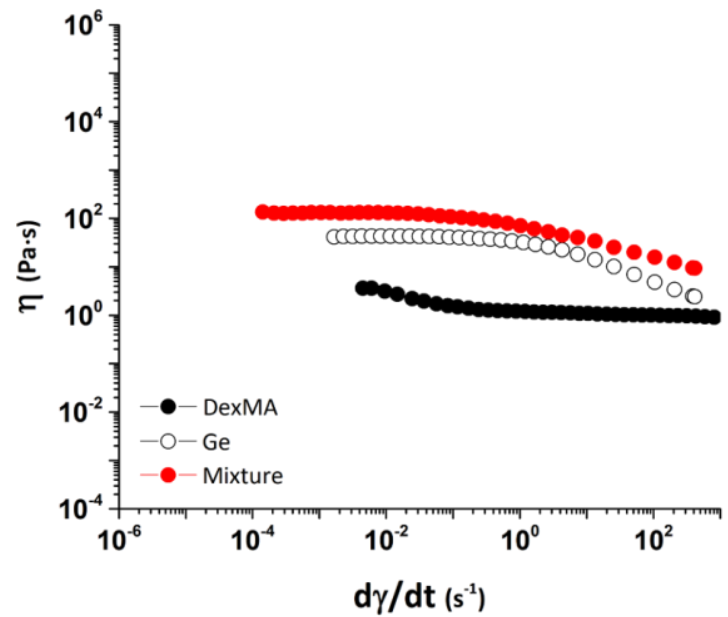

$0.25 \% \mathrm{Ge}$

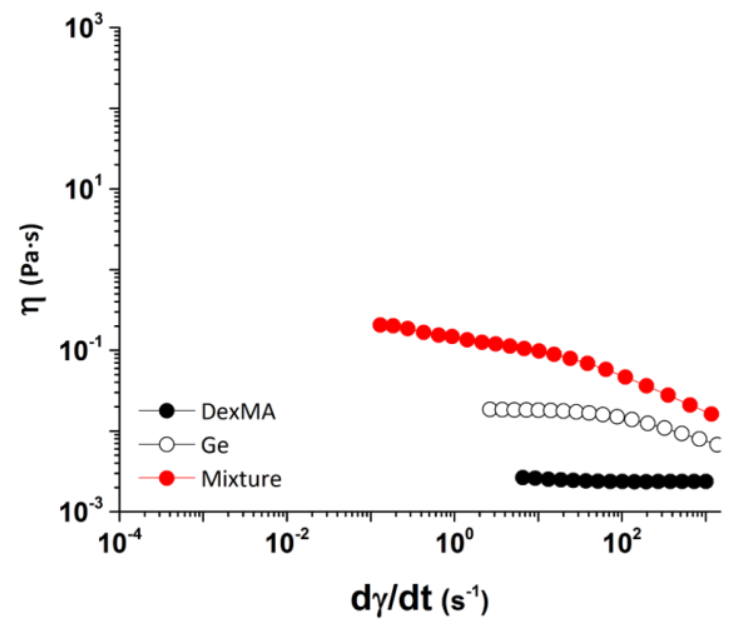

$0.75 \% \mathrm{Ge}$

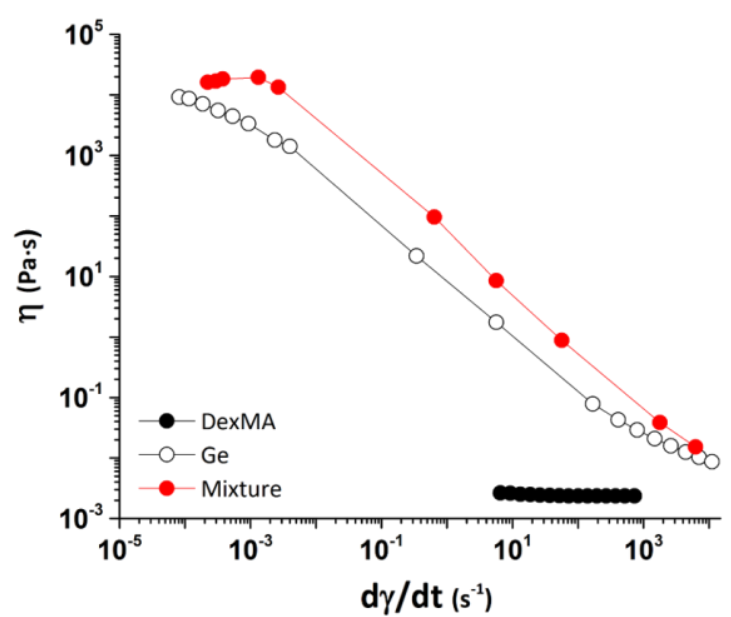

$2 \% \mathrm{Ge}$

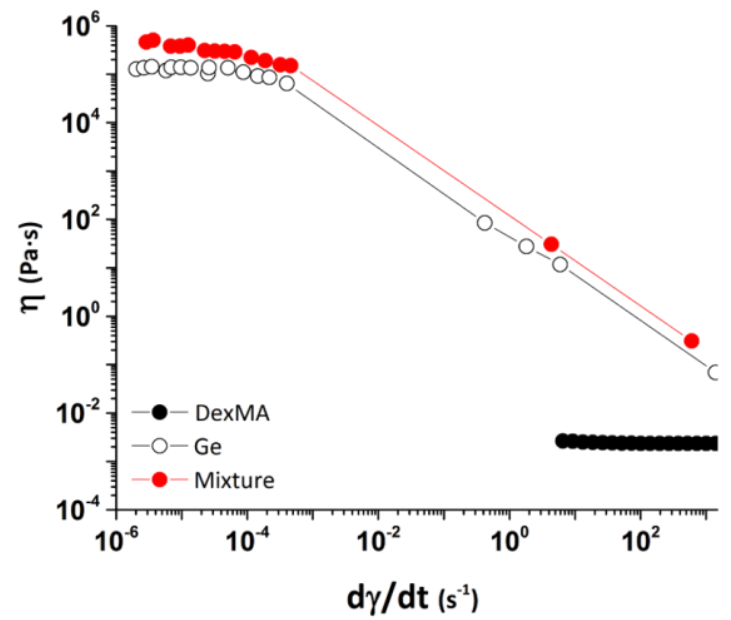

Fig. 7 Viscosity curves of 5\% DexMA, Ge $(0.25$ to $2 \% \mathrm{w} / \mathrm{w})$ and their mixtures in (left) glycerol and in (right) HEPES: (a) 5\% DexMA/0.25\%Ge; (b) 5\%DexMA/0.75\%Ge; (c) 5\%DexMA/2\%Ge. 
Flow curves, reported in Fig. 7 (a-c), clearly evidenced that blends of DexMA and Ge showed an increase in the viscosity values in comparison to solutions of the single DexMA and Ge. This increase can be due to a synergistic effect, ascribed to the interpenetrations of the polymer chains. This interpenetration occurred not only in HEPES, but also in glycerol. However, blends in glycerol were more homogeneous and differently structured compared to blends in HEPES, as shown by Fig. 8 where $\eta$ and $\eta^{*}$ are plotted against the shear rate and frequency. The application of the "semi-empirical" Cox-Merz rule to $\eta$ and $\eta^{*}$ of blends prepared in HEPES, was not satisfactory, whereas a good superimposition was observed for blends prepared in glycerol for all concentrations. This result - especially evident in the range 0.1 to $10 \mathrm{~Hz} / \mathrm{s}^{-1}$ - can be due to the plasticizing capacity of glycerol and to the good mixing among DexMA and Ge chains.

The blends of DexMA and Ge in aqueous media give irregular samples. In fact, Ge chains preserve the ability to form gels above a critical concentration leading to the formation of domains where junction zones of double helix aggregates are present and where DexMA chains are partially excluded, thus leading to the formation of a nonhomogeneous network. 
(a)

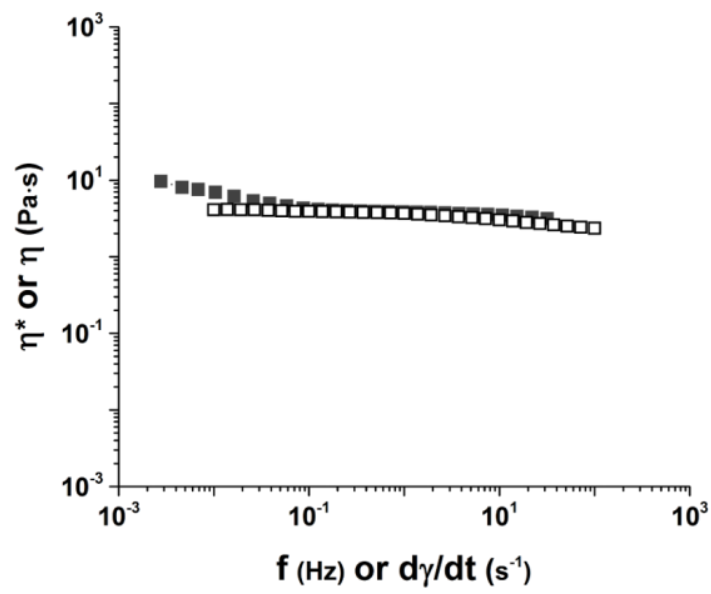

(b)

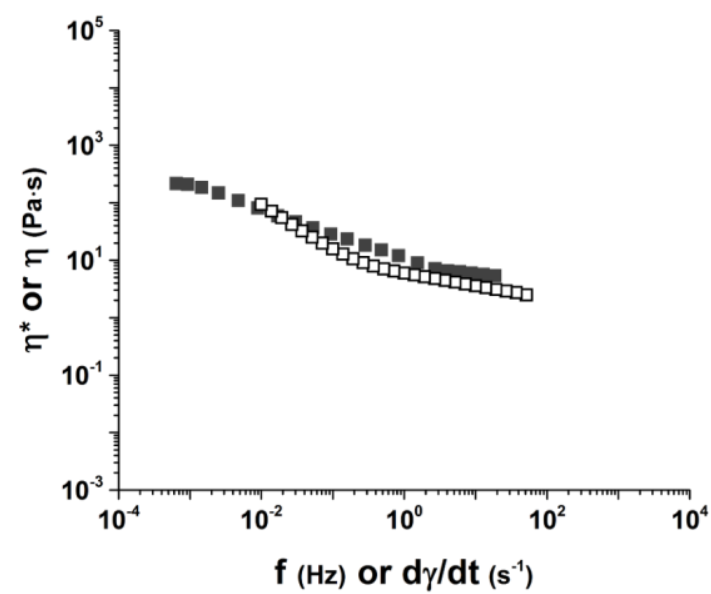

(c)

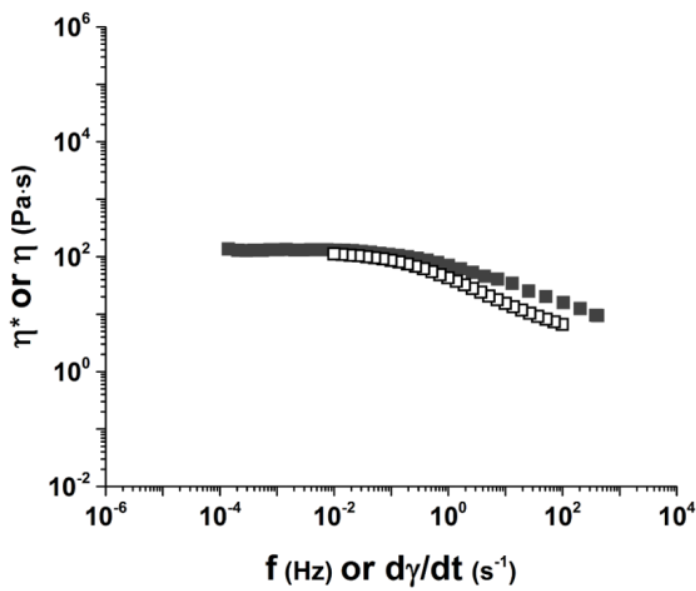

$0.25 \% \mathrm{Ge}$

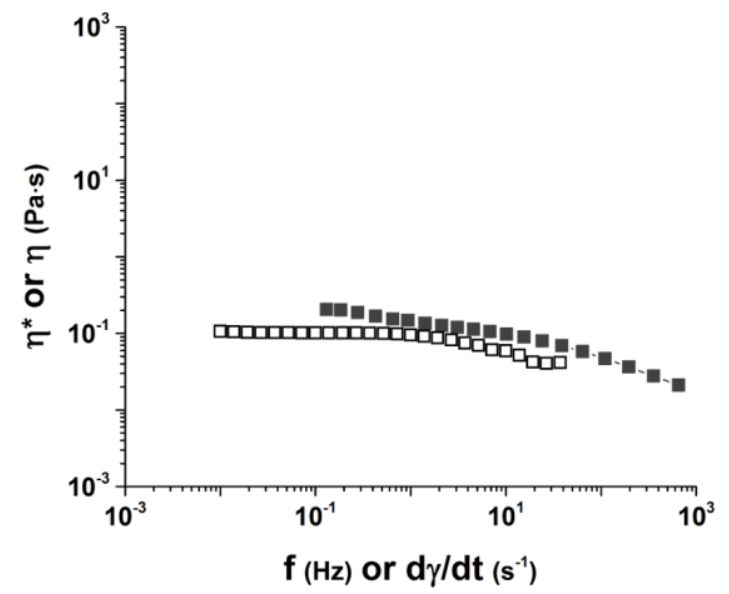

$0.75 \% \mathrm{Ge}$

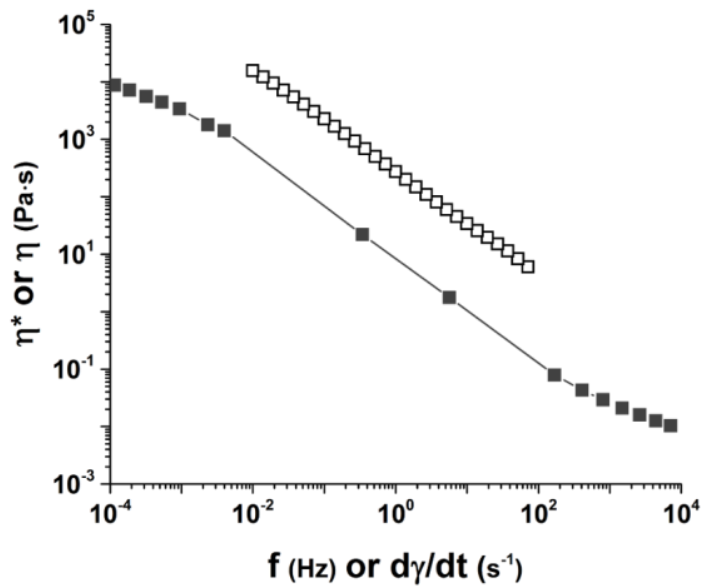

$2 \% \mathrm{Ge}$

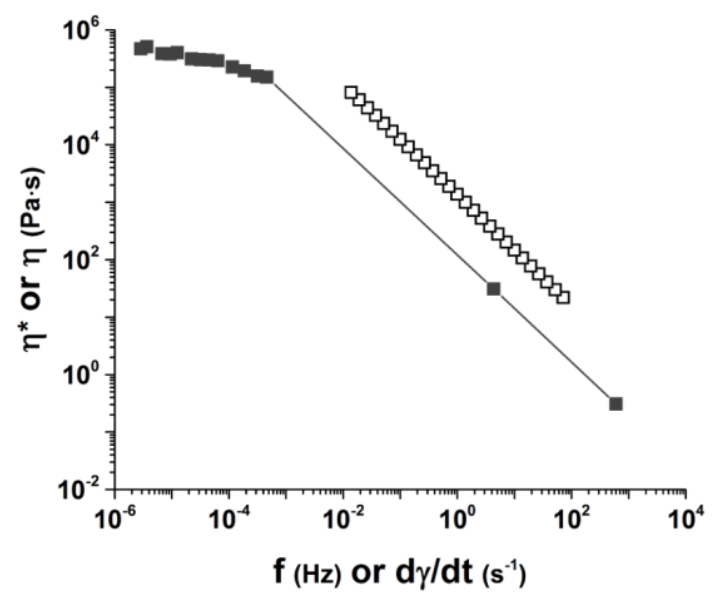

Fig. 8 Steady shear viscosity (full symbols) and complex viscosity (empty symbols) plotted against shear rate and frequency for DexMA/Ge mixtures in glycerol (left) and HEPES (right): 5\%DexMA/0.25\%Ge (a); 5\%DexMA/0.75\%Ge (b); $5 \%$ DexMA $/ 2 \% G e(c)$. 
Subsequently, DexMA/Ge mixtures in HEPES or glycerol were exposed to UV light in the presence of I-2959, with the aim to chemically crosslink the methacrylate moieties of DexMA, leading to the formation of the semi-IPNs (Fig. 5). The kinetic of the crosslinking reaction was investigated for the semi-IPNs prepared both in glycerol and HEPES (Fig. 9a and $9 b$ ). The presence of Ge did not affect the time required for the crosslinking of DexMA chains in both the solvents: the $\mathrm{G}^{\prime}(1 \mathrm{~Hz})$ of the semi-IPNs at $1 \mathrm{~min}$ and after $15 \mathrm{~min}$ in glycerol or HEPES, respectively, is almost the same at all the tested Ge concentrations. After the UV curing, DexMA/Ge scaffolds were investigated through rheology and dynamomechanical analyses to assess the effect of Ge concentration on DexMA networks. Fig. 9c and 9d show the mechanical spectra of the semi-IPNs which are compared with those of the DexMA network, confirming that the elastic properties of the semi-IPNs were dominated by the DexMA and the presence of Ge did not interfere with the DexMA network formation. In both solvents, the systems behave as a gel with $G^{\prime}>G^{\prime \prime}$. However, by comparing the mechanical spectra of the networks in pure glycerol with those prepared in HEPES, it was evidenced that DexMA network formation in HEPES is affected by Ge, only at high Ge concentration: $G^{\prime}$ values are higher more than one order of magnitude when $2 \% \mathrm{w} / \mathrm{w}$ Ge was used for the formation of the semi-IPN. Furthermore, in glycerol G" values are frequency dependent. This behaviour can be ascribed to the plasticity induced by the glycerol to the network.

(a)

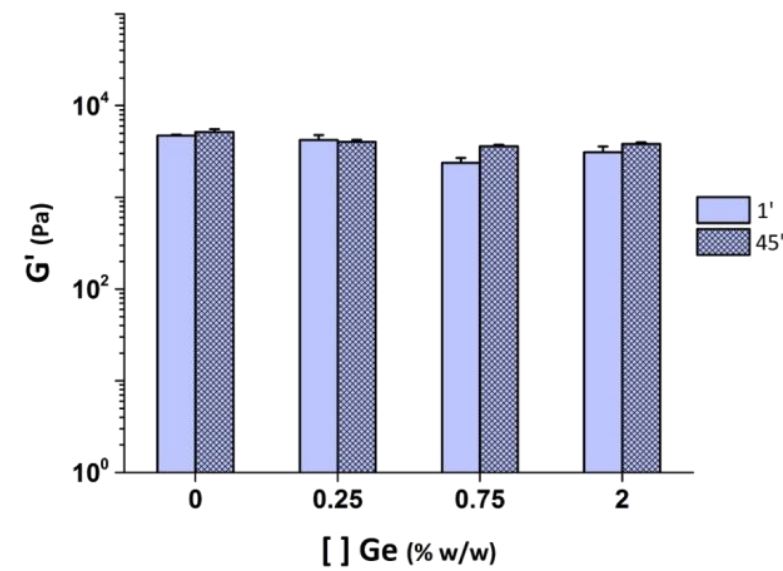

(c)

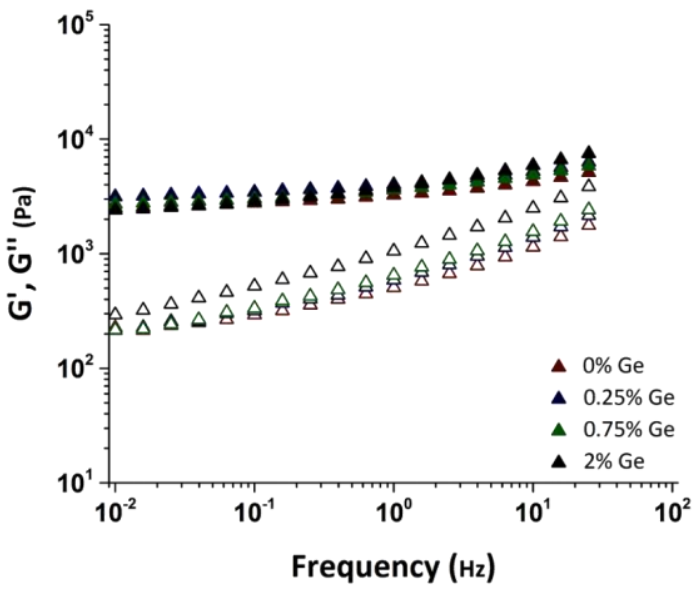

(b)

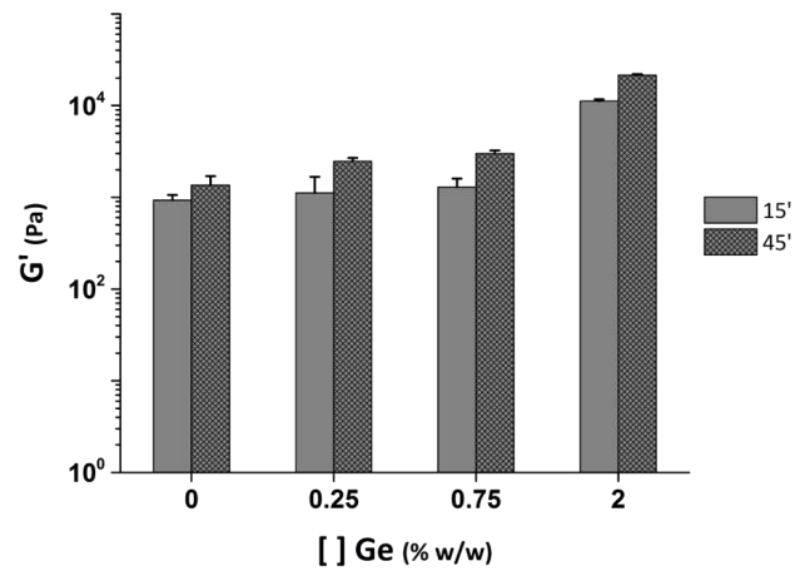

(d)

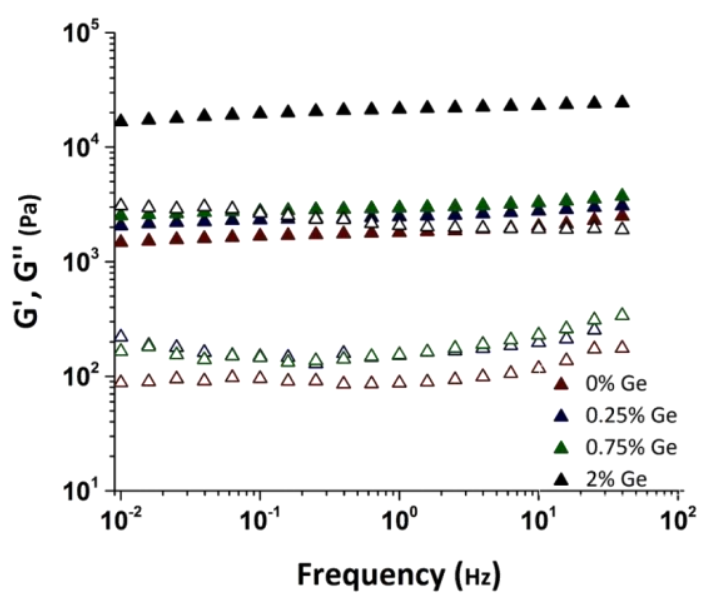

Fig. 9 Storage modulus, $\mathrm{G}^{\prime}$, at $1 \mathrm{~Hz}$, as a function of Ge concentration (ranging from 0 to $2 \% \mathrm{w} / \mathrm{w}$ ) for $5 \% \mathrm{w} / \mathrm{w}$ DexMA/Ge networks prepared in pure glycerol (light blue bars) (a) and HEPES (grey bars) (b) as solvents. Storage 
modulus G' (filled symbols) and loss modulus G" (open symbols) of 5\% w/w DexMA gel and 5\%DexMA/0.25\%Ge, 5\%DexMA/0.75\%Ge and 5\%DexMA/2\%Ge semi-IPNs, as a function of the frequency, in glycerol (c) and in HEPES (d).

Dynamo-mechanical analyses were also performed on DexMA and DexMA/Ge networks prepared in both glycerol and HEPES. The ultimate strain and stress values of HEPES-based gels were lower than those of glycerol-based systems. The rupture of the gels occurred at $60 \%$ of deformation in glycerol and at lower deformation in HEPES, while the compressive stress was one order of magnitude higher in glycerol than in HEPES (Fig. 10). Also the effect of the Ge concentration on the dynamo-mechanical properties of the semi-IPNs was investigated. Specifically, the stress-strain curves are almost superimposable for the lowest concentrations of $\mathrm{Ge}(0.25 \%$ and $0.75 \%)$ in both solvents, while a significant difference occurred only at $2 \% \mathrm{w} / \mathrm{w}$ Ge (Fig. S2 SI a and b). These results indicate that, in glycerol, the gels can be easily deformed before breaking thanks to the glycerol ability to dissipate more efficiently the deformation energy. Moreover, systems in glycerol exhibited higher Young moduli compared to those prepared in HEPES (Fig. 11). By comparing the effect of Ge concentration on the semi-IPNs formulated in HEPES or glycerol, it is evident that the Young moduli increase with the increase of the polymer concentration (except for the 5\%DexMA/2\%Ge semi-IPN in glycerol). Therefore, dynamo-mechanical analyses indicate that both glycerol and Ge enhanced the mechanical properties (e.g. elasticity and stiffness) of the semi-IPNs, showing a synergistic effect compared to the same systems prepared in HEPES. 
(a)

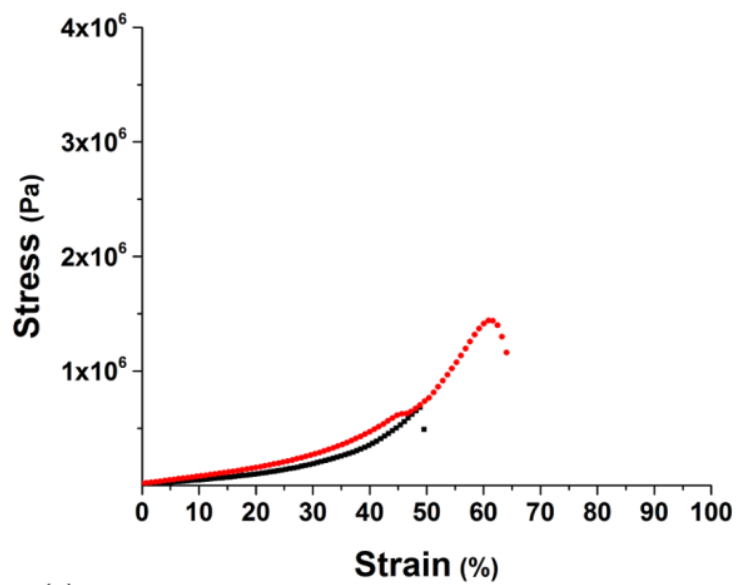

(c)

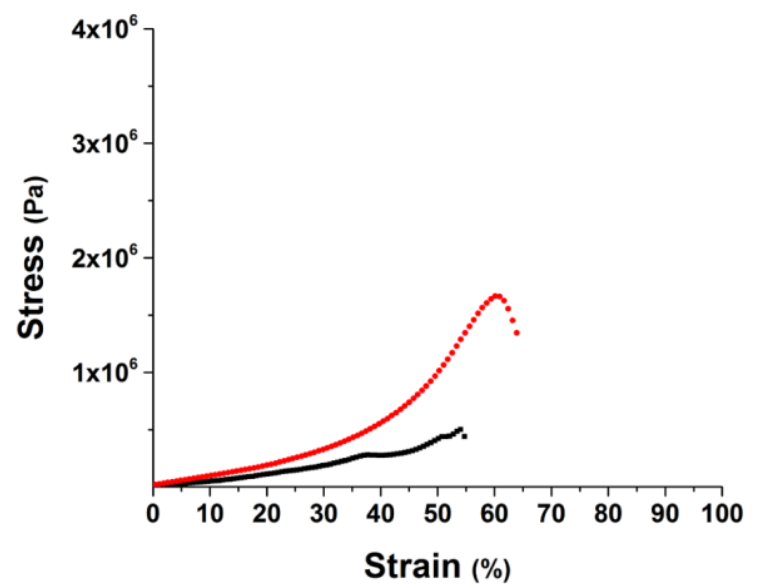

(b)

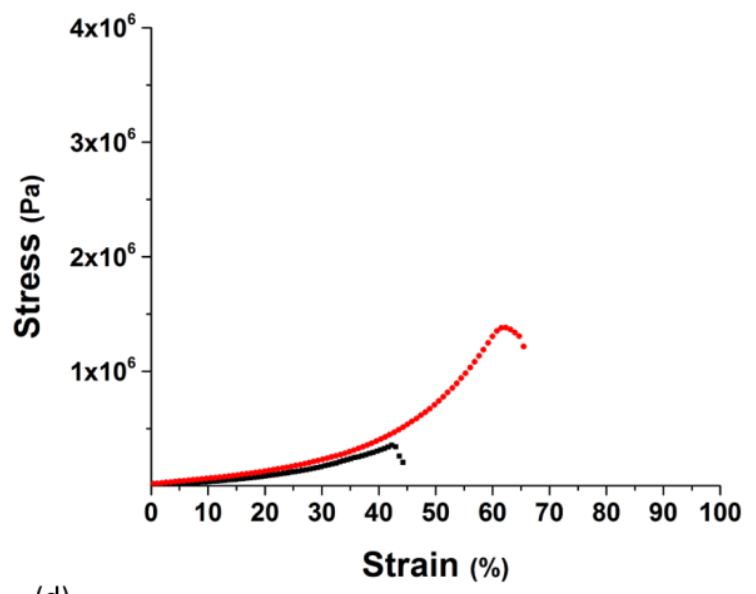

(d)

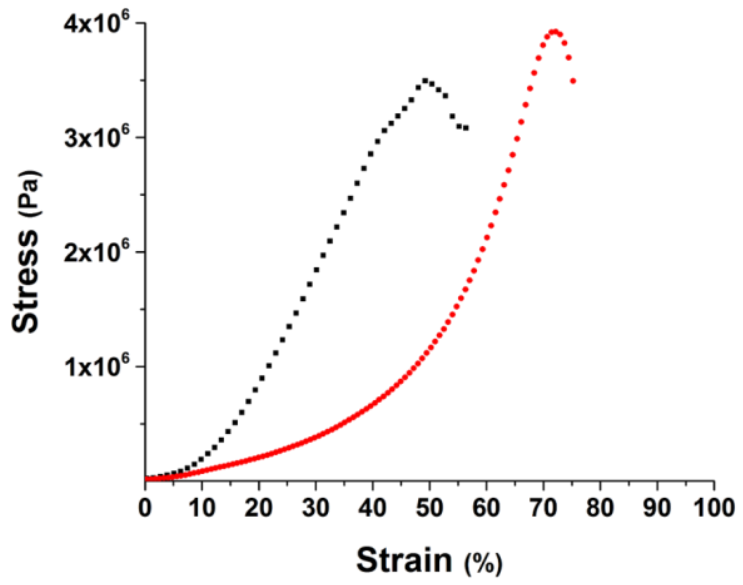

Fig. 10 Stress-strain plots of DexMA gel and DexMA/Ge semi-IPNs in HEPES (black) and glycerol (red): (a) 5\%DexMA;

(b) 5\%DexMA/0.25\%Ge; (c) 5\%DexMA/0.75\%Ge; (d) 5\%DexMA/2\%Ge.

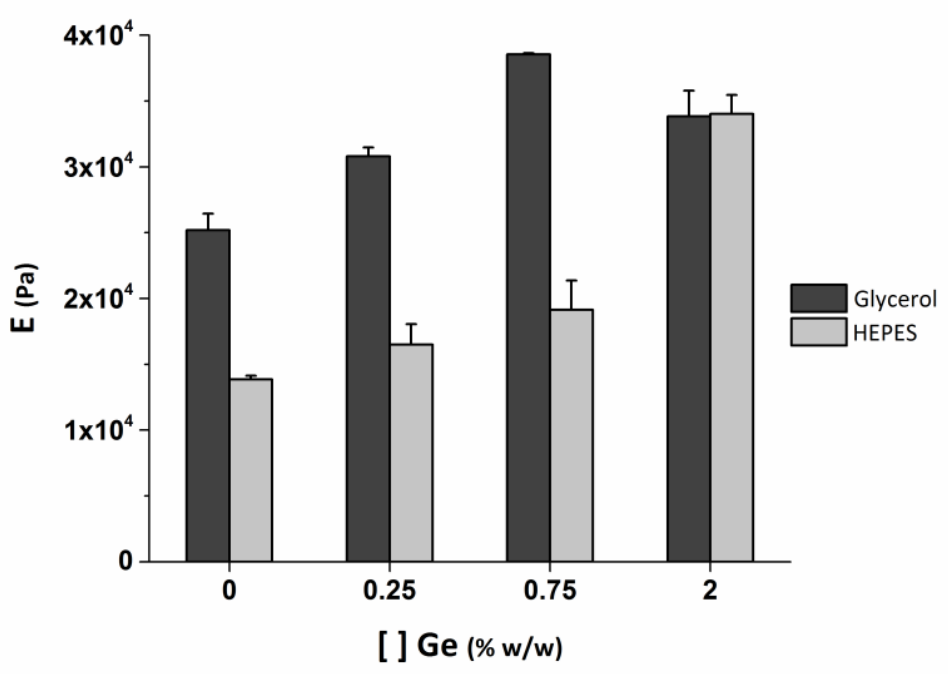

Fig. 11 Young moduli (E) of DexMA gel and DexMA/Ge semi-IPNs prepared in HEPES or glycerol. 


\section{Conclusions}

In this work, glycerol was investigated as a green solvent for the development of DexMA/Ge semi-IPNs. Substitution of water with glycerol clearly led to an enhancement of both the formation process and properties of these scaffolds. In detail, glycerol is responsible for a faster crosslinking kinetic of DexMA, leading to the formation of a complete network in less than one minute. This feature is useful in all the applications in which a fast crosslinking kinetic is requested, such as in $3 \mathrm{D}$ printing where a rapid gelation is necessary to maintain the structure immediately after printing. Furthermore, the ability of glycerol to lead to well-defined porous structures of DexMA gly-semi-IPNs may be particularly interesting in tissue engineering, for the encapsulation and culture of mammalian cells, enhancing cell migration through the green synthetic porous network. Finally, the switching of water to glycerol leads to the formation of transparent scaffolds that can be useful as cell culture platforms, thanks to their capability to effectively tackle the limitations of current 3D optical imaging (i.e. visibility, spatial resolution, and penetration depth). Anyhow, the advantages of using glycerol as a solvent are not limited to DexMA/Ge semi-IPNs. In fact, several polymers can be easily solubilized in glycerol and their functionalization with methacrylate moieties can be investigated to obtain faster crosslinking kinetics with a rapid formation of the scaffolds. Furthermore, the ability of glycerol to reduce the viscosity of $\mathrm{Ge}$ solutions and to enhance the homogeneity of their mixtures with other polymers (e.g. DexMA) could be further investigated for the formation of innovative IPNs that cannot be obtained in aqueous media. To conclude, this work clearly shows the key role of glycerol as a green solvent for the development of new scaffolds with a wide range of applications, such as 3D printing and biomedicine.

\section{Conflicts of interest}

There are no conflicts to declare.

\section{Compliance with ethical standards}

This manuscript does not contain any studies with animals performed by any of the authors.

\section{Funding}

The authors acknowledge financial support from Sapienza University of Rome ("Finanziamenti di Ateneo per la Ricerca Scientifica - RP116154C2EF9AC8 and RM11715C1743EE89").

\section{Acknowledgments}

Authors thank Ezio Battaglione (Section of Human Anatomy, Electron Microscopy Laboratory "Pietro M Motta", University of Rome "Sapienza") for his support in electron microscopy experiments.

\section{Author contributions}

Data curation, Tommasina Coviello and Stefania Petralito; Funding acquisition, Pietro Matricardi and Giuseppe Familiari; Investigation, Nicole Zoratto and Roberto Matassa; Methodology, Nicole Zoratto and Elita Montanari; Project administration, Chiara Di Meo and Pietro Matricardi; Writing-original draft, Nicole Zoratto; Writing-review and editing, Tommasina Coviello and Elita Montanari.

\section{Supplementary Informations:}

The online version of this article contains supplementary material, which is available to authorized users. 


\section{References:}

1. The Soap and Detergent Association (1990). Glycerine an overview. 475 Park Avenue South, New York.

2. A. Farrán, C. Cai, M. Sandoval, Y. Xu, J. Liu, M. J. Hernáiz \& R. J. Linhardt (2015). Green solvents in carbohydrate chemistry: From raw materials to fine chemicals. Chemical reviews 115:6811-6853.

3. Y. Gu, J. Barrault \& F. Jérôme (2008). Glycerol as an efficient promoting medium for organic reactions. Advanced Synthesis and Catalysis 350:2007-2012.

4. A. E. Díaz-Álvarez, J. Francos, P. Croche \& V. Cadierno (2014). Recent advances in the use of glycerol as green solvent for synthetic organic chemistry. Current Green Chemistry 1:51-65.

5. J. I. Garcia, H. Garcia-Marin \& E. Pires (2014). Glycerol based solvents: synthesis, properties and applications. Green Chemistry 16:1007-1033.

6. B. S. Fernandes, J. Carlos Pinto, E. C. Cabral-Albuquerque \& R. L. Fialho (2015). Free-Radical Polymerization of Urea, Acrylic Acid, and Glycerol in Aqueous Solutions. Polymer Engineering \& Science 55: 1219-1229.

7. T. O. Osmanov, N. V. Kozlova \& V. Y. Markov (1990). Polymerization of acrylamide in water-glycerol mixtures. Polymer Science U.S.S.R. 32:650-654.

8. B. C. Chun, M. H. Chong \& Y. C. Chung (2007). Effect of glycerol cross-linking and hard segment content on the shape memory property of polyurethane block copolymer. Journal of materials science, 42:6524-6531.

9. M. A. Cerqueira, B. W. Souza, J. A. Teixeira \& A. A. Vicente (2012). Effect of glycerol and corn oil on physicochemical properties of polysaccharide films-A comparative study. Food Hydrocolloids 27:175-184.

10. M. G. A. Vieira, M. A. Da Silva, L. O. Dos Santos \& M. M. Beppu (2011). Natural-based plasticizers and biopolymer films: A review. European Polymer Journal 47:254-263.

11. P. Paolicelli, S. Petralito, G. Varani, M. Nardoni, S. Pacelli, L. Di Muzio, J. Tirillò, C. Bartuli, S. Cesa, M. A. Casadei \& A. Adrover (2018). Effect of glycerol on the physical and mechanical properties of thin gellan gum films for oral drug delivery. International Journal of Pharmaceutics, 547:226-234.

12. E. I. Stout \& A. McKessor (2012). Glycerin-Based Hydrogel for Infection Control. Advances in wound care 1:48-51.

13. S. Björklund, J. Engblom, K. Thuresson \& E. Sparr (2013). Glycerol and urea can be used to increase skin permeability in reduced hydration conditions. European Journal of Pharmaceutical Sciences 50:638-645.

14. M. Cassanelli, I. Norton \& T. Mills (2017). Effect of alcohols on gellan gum gel structure: Bridging the molecular level and the three-dimensional network. Food Structure 14:112-120.

15. Z. Q. Liu, X. S. Yi \& Y. Feng (2001). Effects of glycerin and glycerol monostearate on performance of thermoplastic starch. Journal of materials science 36:1809-1815.

16. M. Q. Guo, X. Hu, C. Wang \& L. Ai (2017). Polysaccharides: structure and solubility. In. "Solubility of polysaccharides", pag. 7-21.

17. R. Häkkinen \& A. Abbott (2019). Solvation of carbohydrates in five choline chloride-based deep eutectic solvents and the implication for cellulose solubility. Green Chemistry 21:4673-4682.

18. A. D. Jenkins, P. Kratochvíl, R. F. T. Stepto \& U. W. Suter (1996). Glossary of basic terms in polymer science (IUPAC Recommendations 1996). Pure and applied chemistry 68:2287-2311.

19. X. Qi, W. Wei, J. Li, Y. Liu, X. Hu, J. Zhang, B. Lirong \& W. Dong (2015). Fabrication and characterization of a novel anticancer drug delivery system: salecan/poly (methacrylic acid) semi-interpenetrating polymer network hydrogel. ACS Biomaterials Science \& Engineering 1:1287-1299.

20. X. Qi, W. Wei, J. Li, T. Su, X. Pan, G. Zuo, Zhang J. \& W. Dong (2017). Design of Salecan-containing semi-IPN hydrogel for amoxicillin delivery. Materials Science and Engineering: C 75:487-494. 
21. X. Qi, Y. Yuan, J. Zhang, J. W. Bulte \& W. Dong (2018). Oral administration of salecan-based hydrogels for controlled insulin delivery. Journal of Agricultural and Food Chemistry 66:10479-10489.

22. P. Matricardi, M. Pontoriero, T. Coviello, M. A. Casadei \& F. Alhaique (2008). In situ cross-linkable novel alginatedextran methacrylate IPN Hydrogels for biomedical applications: mechanical characterization and drug delivery properties. Biomacromolecules 9:2014-2020.

23. L. Pescosolido, W. Schuurman, J. Malda, P. Matricardi, F. Alhaique, T. Coviello, P. R. Van Weeren, W. J. A. Dhert, W. E. Hennink \& T. Vermonden (2011). Hyaluronic acid and dextran-based semi-IPN hydrogels as biomaterials for bioprinting. Biomacromolecules 12:1831-1838.

24. D. Bellini, C. Cencetti, J. Meraner, D. Stoppoloni, A. Scotto D’Abusco \& P. Matricardi (2015). An in situ gelling system for bone regeneration of osteochondral defects. European Polymer Journal 72:642-650.

25. L. Pescosolido, T. Vermonden, J. Malda, R. Censi, W. J. Dhert, F. Alhaique, W. E. Hennink \& Matricardi, P. (2011). In situ forming IPN hydrogels of calcium alginate and dextran-HEMA for biomedical applications. Acta biomaterialia 7:1627-1633.

26. W. N. E. Van Dijk-Wolthuis, O. Franssen, H. Talsma, M. J. van Steenbergen, J. J. Kettenes-van den Bosh \& W. E. Hennink (1995). Synthesis, characterization, and polymerization of glycidyl methacrylate derivatized dextran. Macromolecules 28:6317-6322.

27. S. C. De Smedt, A. Lauwers, J. Demeester, M. J. Van Steenberg, W. E. Hennink \& S. P. F. M. Roefs (1995). Characterization of the network structure of dextran glycidyl methacrylate hydrogels by studying the rheological and swelling behaviour. Macromolecules 28:5082-5088.

28. A. Amici, G. Caracciolo, L. Digiacomo, V. Gambini, C. Marchini, M. Tilio, A. L. Capriotti, V. Colapicchioni, R. Matassa, G. Familiari, S. Palchetti, D. Pozzi, M. Mahmoudi \& A. Lagan (2017). In vivo protein corona patterns of lipid nanoparticles. RSC Advances 7:1137-1145.

29. E. Antoniou, M. Tsianou \& P. Alexandridis (2018). Solvent modulation of polysacharide conformation. In AIChE Annual Meeting, Conference Proceedings.

30. E. Miyoshi \& K. Nishinari (1999). Non-Newtonian flow behaviour of gellan gum aqueous solutions. Colloid and Polymer Science 277:727-734.

31. M. Dentini, T. Coviello, W. Burchard \& V. Crescenzi (1998). Solution properties of exocellular microbial polysaccharides. 3. Light scattering from gellan and from the exocellular polysaccharide of Rhizobium trifolii (strain TA1) in the ordered state. Macromolecules, 21:3312-3320.

32. L. Yang \& A. T. Paulson (2000). Mechanical and water vapour barrier properties of edible gellan films. Food Research International 33:563-570.

33. S. Zhao, Z. Shen, J. Wang, X. Li, Y. Zeng, B. Wang, Y. He, \& Y. Du (2014). Glycerol-mediated nanostructure modification leading to improved transparency of porous polymeric scaffolds for high performance 3D cell imaging. Biomacromolecules 15:2521-2531.

34. R. Matassa, S. Orlanducci, V. Guglielmotti, D. Sordi, E. Tamburri, M. L. Terranova, M. Passeri \& D. Rossi (2014). Characterization of Carbon Structures produced by Graphene Self-Assembling. Journal of Applied Crystallography 47:222-227.

35. R. Matassa, G. Familiari, E. Battaglione, C. Sibilia, G. Leahu, A. Belardini, I. Venditti, L. Fontana \& I. Fratoddi (2016). Electron Microscopy Reveals Layered Architecture of Individual Gold Nanoparticles Self-Anchored by Fluorescence Monomers. Nanoscale 8:18161-18169. 


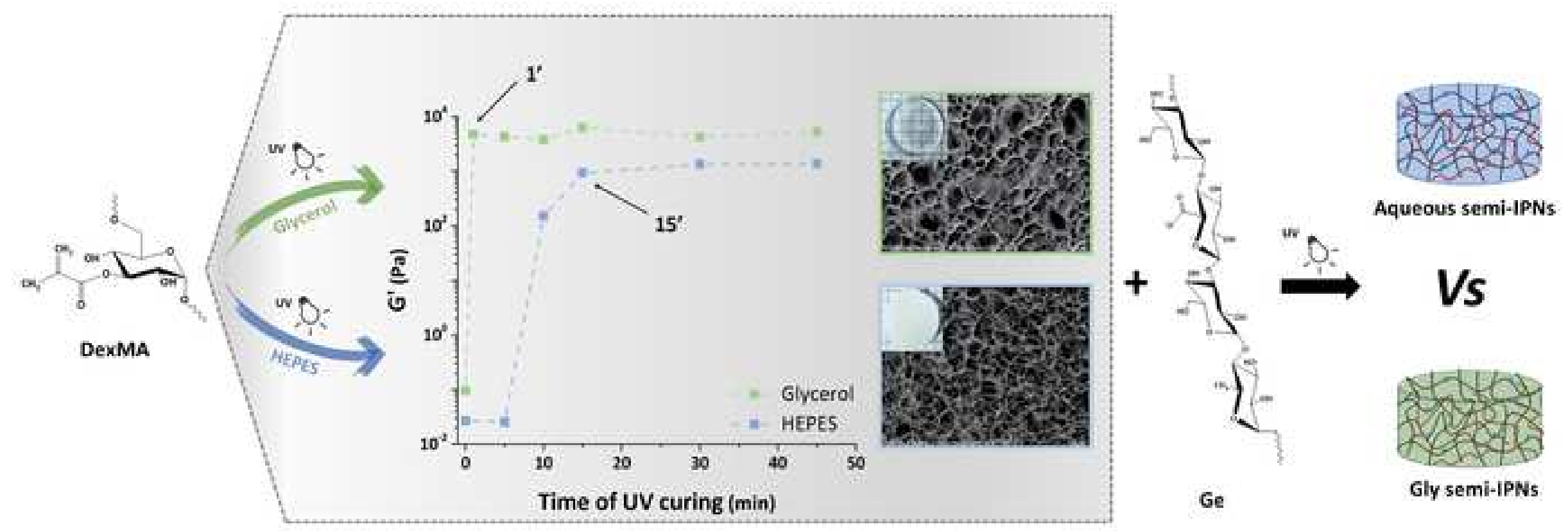


Click here to access/download Supplementary Material SI_Matricardi.docx 